\title{
QCD phase diagram at finite baryon and isospin chemical potentials
}

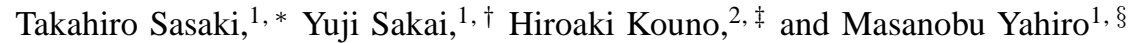 \\ ${ }^{1}$ Department of Physics, Graduate School of Sciences, Kyushu University, Fukuoka 812-8581, Japan \\ ${ }^{2}$ Department of Physics, Saga University, Saga 840-8502, Japan
}

(Dated: November 1, 2018)

\begin{abstract}
The phase structure of two-flavor QCD is explored for thermal systems with finite baryon- and isospinchemical potentials, $\mu_{\mathrm{B}}$ and $\mu_{\text {iso }}$, by using the Polyakov-loop extended Nambu-Jona-Lasinio (PNJL) model. The PNJL model with the scalar-type eight-quark interaction can reproduce lattice QCD data at not only $\mu_{\text {iso }}=$ $\mu_{\mathrm{B}}=0$ but also $\mu_{\text {iso }}>0$ and $\mu_{\mathrm{B}}=0$. In the $\mu_{\text {iso }}-\mu_{\mathrm{B}}-T$ space, where $T$ is temperature, the critical endpoint of the chiral phase transition in the $\mu_{\mathrm{B}}-T$ plane at $\mu_{\mathrm{iso}}=0$ moves to the tricritical point of the pion-superfluidity phase transition in the $\mu_{\text {iso }}-T$ plane at $\mu_{\mathrm{B}}=0$ as $\mu_{\text {iso }}$ increases. The thermodynamics at small $T$ is controlled by $\sqrt{\sigma^{2}+\pi^{2}}$ defined by the chiral and pion condensates, $\sigma$ and $\pi$.

PACS numbers: 11.30.Rd, 12.40.-y
\end{abstract}

\section{INTRODUCTION}

The phase diagram of quantum chromodynamics $(\mathrm{QCD})$ is the key to understanding not only natural phenomena such as compact stars and the early Universe but also laboratory experiments such as relativistic heavy-ion collisions. Quantitative calculations of the phase diagram from the first-principle lattice QCD (LQCD) have the well-known sign problem when the baryon chemical potential $\left(\mu_{\mathrm{B}}\right)$ is real [1]; here, $\mu_{\mathrm{B}}$ is related to the quark-number chemical potential $\mu_{\mathrm{q}}$ as $\mu_{\mathrm{B}}=3 \mu_{\mathrm{q}}$. Several approaches have been proposed so far to circumvent the difficulty; for example, the reweighting method [2], the Taylor expansion method [3] and the analytic continuation from imaginary $\mu_{\mathrm{q}}$ to real $\mu_{\mathrm{q}}[4-6]$. However, those are still far from perfection particularly at $\mu_{\mathrm{q}} / T \gtrsim 1$, where $T$ is temperature.

As an approach complementary to LQCD, we can consider effective models such as the Nambu-Jona-Lasinio (NJL) model [7-12] and the Polyakov-loop extended NambuJona-Lasinio (PNJL) model [13-33]. The NJL model describes the chiral symmetry breaking, but not the confinement mechanism. The PNJL model is extended so as to treat both the mechanisms [14] approximately by considering the Polyakov-loop in addition to the chiral condensate as ingredients of the model.

In the NJL-type models, the input parameters are usually determined from the pion mass and the pion decay constant at vacuum $\left(\mu_{\mathrm{q}}=0\right.$ and $\left.T=0\right)$. Some of the models have the scalar-type eight-quark interaction. The strength of the interaction is adjusted to LQCD data at finite $T$ [31], since the sigma-meson mass at vacuum related to the interaction has a large error bar and then ambiguous [34]. It is then highly nontrivial whether the models predict properly dynamics of QCD at finite $\mu_{\mathrm{q}}$. This should be tested from QCD. Fortunately, this is possible at imaginary $\mu_{\mathrm{q}}$, since LQCD has no

\footnotetext{
*sasaki@phys.kyushu-u.ac.jp

† sakai@phys.kyushu-u.ac.jp

łkounoh@cc.saga-u.ac.jp

$\S$ yahiro@phys.kyushu-u.ac.jp
}

sign problem there. In Ref. [32], it was shown that the PNJL model can reproduce LQCD data at imaginary $\mu_{\mathrm{q}}$. QCD has the Roberge-Weiss periodicity and the Roberge-Weiss transition [35] in the imaginary $\mu_{\mathrm{q}}$ region, because of the extended $\mathbb{Z}_{3}$ symmetry [31-33]. The PNJL model can reproduce these, since it has the symmetry [31]. In the real $\mu_{\mathrm{q}}$ region, as an important result, a phase diagram is predicted by the PNJL model with the parameter set [32] determined from the LQCD data at imaginary $\mu_{\mathrm{q}}$. The PNJL prediction shows that in the $\mu_{\mathrm{q}}-T$ plane at $\mu_{\mathrm{I}}=0$ there appears a critical endpoint (CEP), that is, a second-order critical point where a first-order chiral phase-transition line terminates.

A similar test of the PNJL model is possible for finite isospin-chemical potential $\left(\mu_{\text {iso }}\right)$ [36], since LQCD has no sign problem there; for later convenience, we use the "modified" isospin-chemical potential $\mu_{\mathrm{I}}=\mu_{\text {iso }} / 2$ instead of $\mu_{\text {iso }}$. LQCD data are available for both real [37] and imaginary [38, 39] $\mu_{\text {iso. The PNJL model has already been applied }}$ to the real $\mu_{\mathrm{I}}[23,24]$ and the imaginary $\mu_{\mathrm{I}}$ case [40] with success in reproducing the LQCD data. The PNJL calculation at real $\mu_{\mathrm{I}}$ shows that in the $\mu_{\mathrm{I}}-T$ plane at $\mu_{\mathrm{q}}=0$ there exists a first-order pion-superfluidity phase-transition line connected to a second-order pion-superfluidity phase-transition line; the connecting point is a tricritical point (TCP) by definition. Real $\mu_{\mathrm{I}}$ dependence of QCD phase diagram is investigated in other models such as chiral perturbation theory [36], the strong coupling QCD [41] and so on [42].

The CEP in the $\mu_{\mathrm{q}}-T$ plane at $\mu_{\mathrm{I}}=0$ is important as a good indicator of the chiral and deconfinement phase transitions in collider experiments at Helmholtzzentrum für Schwerionenforschung GmbH (GSI), Super Proton Synchrotron (SPS) [43, 44], Relativistic Heavy Ion Collider (RHIC) [45, 46] and LHC [47]. In the measurements, $\mu_{\mathrm{I}}$ is not zero generally. It is then interesting to see how critical points such as CEP and TCP are located in the $\mu_{\mathrm{q}}-\mu_{\mathrm{I}}-T$ space.

In this paper, we draw the phase diagram of two-flavor QCD in the $\mu_{\mathrm{I}}-\mu_{\mathrm{q}}-T$ space by using the PNJL model. Following our previous paper [32], we introduce the scalar-type eight-quark interaction to reproduce LQCD data on thermal systems at $\mu_{\mathrm{q}}=\mu_{\mathrm{I}}=0$. The scalar-type eight-quark interaction is a next-to-leading order correction in the power counting rule based on mass dimension. First, we will show that 
the PNJL model with the parameter set thus determined also reproduces LQCD data on thermal systems at $\mu_{\mathrm{I}}>0$ and $\mu_{\mathrm{q}}=0$. After confirming the reliability of the present PNJL model, we will predict locations of CEP and TCP in the $\mu_{\mathrm{I}^{-}}$ $\mu_{\mathrm{q}}-T$ space.

In Sec. II the PNJL model is recapitulated. In Sec. III, the PNJL calculation is compared with LQCD data for thermal systems at $\mu_{\mathrm{I}}>0$ and $\mu_{\mathrm{q}}=0$, and the phase diagram is explored in the $\mu_{\mathrm{I}}-\mu_{\mathrm{q}}-T$ space. Properties of the susceptibilities near CEP and TCP are analyzed. Section IV is devoted to a summary.

\section{PNJL MODEL}

The two-flavor PNJL Lagrangian in Euclidean space-time is

$$
\begin{aligned}
\mathcal{L} & =\bar{q}\left(\gamma_{\nu} D^{\nu}-\gamma_{4} \hat{\mu}+\hat{m}_{0}\right) q+G_{\mathrm{s}}\left[(\bar{q} q)^{2}+\left(\bar{q} i \gamma_{5} \vec{\tau} q\right)^{2}\right] \\
& +G_{8}\left[(\bar{q} q)^{2}+\left(\bar{q} i \gamma_{5} \vec{\tau} q\right)^{2}\right]^{2}-\mathcal{U}\left(\Phi[A], \Phi[A]^{*}, T\right),
\end{aligned}
$$

where $D^{\nu}=\partial^{\nu}+i A^{\nu}$ and $A^{\nu}=\delta_{0}^{\nu} g A_{a}^{0} \frac{\lambda^{a}}{2}$ with the gauge field $A_{a}^{\nu}$, the Gell-Mann matrix $\lambda_{a}$ and the gauge coupling $g$. In the NJL sector, $G_{\mathrm{S}}$ denotes the coupling constant of the scalar-type four-quark interaction and $G_{8}$ stands for that of the scalar-type eight-quark interaction [32, 48, 49]. The Polyakov-potential $\mathcal{U}$, defined in (15), is a function of the Polyakov-loop $\Phi$ and its Hermitian conjugate $\Phi^{*}$.

The chemical potential matrix $\hat{\mu}$ is defined by $\hat{\mu}=$ $\operatorname{diag}\left(\mu_{u}, \mu_{d}\right)$ with the $u$-quark ( $d$-quark) number chemical potential $\mu_{u}\left(\mu_{d}\right)$, while $\hat{m}_{0}=\operatorname{diag}\left(m_{0}, m_{0}\right)$. This is equivalent to introducing the baryon and isospin-chemical potentials, $\mu_{\mathrm{B}}$ and $\mu_{\text {iso }}$, coupled, respectively, to the baryon charge $\bar{B}$ and to the isospin charge $\bar{I}_{3}$ :

$$
\hat{\mu}=\mu_{\mathrm{q}} \tau_{0}+\mu_{\mathrm{I}} \tau_{3}
$$

with

$$
\mu_{\mathrm{q}}=\frac{\mu_{u}+\mu_{d}}{2}=\frac{\mu_{\mathrm{B}}}{3}, \mu_{\mathrm{I}}=\frac{\mu_{u}-\mu_{d}}{2}=\frac{\mu_{\text {iso }}}{2},
$$

where $\tau_{0}$ is the unit matrix and $\tau_{i}(i=1,2,3)$ are the Pauli matrices in flavor space. Note that $\mu_{\mathrm{q}}$ is the quark chemical potential and $\mu_{\mathrm{I}}$ is half the isospin-chemical potential $\left(\mu_{\text {iso }}\right)$. In the limit of $m_{0}=\mu_{\mathrm{I}}=0$, the PNJL Lagrangian has the $S U_{\mathrm{L}}(2) \times S U_{\mathrm{R}}(2) \times U_{\mathrm{v}}(1) \times S U_{\mathrm{c}}(3)$ symmetry. For $m_{0} \neq 0$ and $\mu_{\mathrm{I}} \neq 0$, it is reduced to $U_{\mathrm{I}_{3}}(1) \times U_{\mathrm{v}}(1) \times S U_{\mathrm{c}}(3)$.

The Polyakov-loop operator $\hat{\Phi}$ and its Hermitian conjugate $\hat{\Phi}^{\dagger}$ are defined as

$$
\hat{\Phi}=\frac{1}{N} \operatorname{Tr} L, \quad \hat{\Phi}^{\dagger}=\frac{1}{N} \operatorname{Tr} L^{\dagger},
$$

with

$$
L(\mathbf{x})=\mathcal{P} \exp \left[i \int_{0}^{\beta} d \tau A_{4}(\mathbf{x}, \tau)\right],
$$

where $\mathcal{P}$ is the path ordering and $A_{4}=i A^{0}$. In the PNJL model, the vacuum expectation values, $\Phi=\langle\hat{\Phi}\rangle$ and $\Phi^{*}=$
$\left\langle\hat{\Phi}^{\dagger}\right\rangle$, are treated as classical variables. In the Polyakov gauge, $L$ can be written in a diagonal form in color space [14]:

$$
L=e^{i \beta\left(\phi_{3} \lambda_{3}+\phi_{8} \lambda_{8}\right)}=\operatorname{diag}\left(e^{i \beta \phi_{a}}, e^{i \beta \phi_{b}}, e^{i \beta \phi_{c}}\right),
$$

where $\phi_{a}=\phi_{3}+\phi_{8} / \sqrt{3}, \phi_{b}=-\phi_{3}+\phi_{8} / \sqrt{3}$ and $\phi_{c}=$ $-\left(\phi_{a}+\phi_{b}\right)=-2 \phi_{8} / \sqrt{3}$.

The Polyakov-loop $\Phi$ is an exact order parameter of the spontaneous $\mathbb{Z}_{3}$ symmetry breaking in the pure gauge theory. Although the $\mathbb{Z}_{3}$ symmetry is not exact in the system with dynamical quarks, it still seems to be a good indicator of the deconfinement phase transition. Therefore, we use $\Phi$ to define the deconfinement phase transition.

The spontaneous breakings of the chiral and the $U_{\mathrm{I}_{3}}(1)$ symmetry are described by the chiral condensate $\sigma=\langle\bar{q} q\rangle$ and the charged pion condensate [23]

$$
\pi^{ \pm}=\frac{\pi}{\sqrt{2}} e^{ \pm i \varphi}=\left\langle\bar{q} i \gamma_{5} \tau_{ \pm} q\right\rangle,
$$

where $\tau_{ \pm}=\left(\tau_{1} \pm i \tau_{2}\right) / \sqrt{2}$. Since the phase $\varphi$ represents the direction of the $U_{\mathrm{I}_{3}}(1)$ symmetry breaking, we take $\varphi=0$ for convenience. The pion condensate is then expressed by

$$
\pi=\left\langle\bar{q} i \gamma_{5} \tau_{1} q\right\rangle .
$$

Making the mean field (MF) approximation [11, 23], one can obtain the MF Lagrangian as

$$
\begin{aligned}
\mathcal{L}_{\mathrm{MF}}= & \bar{q}\left(\gamma_{\nu} D^{\nu}-\gamma_{4} \hat{\mu}+M \tau_{0}+N i \gamma_{5} \tau_{1}\right) q \\
& -G_{\mathrm{s}}\left[\sigma^{2}+\pi^{2}\right]-3 G_{8}\left(\sigma^{2}+\pi^{2}\right)^{2}-\mathcal{U}
\end{aligned}
$$

with

$$
\begin{aligned}
M & =m_{0}-2 G_{\mathrm{s}} \sigma-4 G_{8} \sigma\left(\sigma^{2}+\pi^{2}\right), \\
N & =-2 G_{\mathrm{s}} \pi-4 G_{8} \pi\left(\sigma^{2}+\pi^{2}\right) .
\end{aligned}
$$

Performing the path integral in the PNJL partition function

$$
Z_{\mathrm{PNJL}}=\int D q D \bar{q} \exp \left[-\int d^{4} x \mathcal{L}_{\mathrm{MF}}\right],
$$

we can get the thermodynamic potential $\Omega$ (per unit volume),

$$
\begin{aligned}
\Omega & =-T \ln \left(Z_{\mathrm{PNJL}}\right) / V=-2 \sum_{i= \pm} \int \frac{d^{3} \mathrm{p}}{(2 \pi)^{3}}\left[3 E_{i}(\mathrm{p})\right. \\
& +\frac{1}{\beta} \ln \left[1+3\left(\Phi+\Phi^{*} e^{-\beta E_{i}^{-}(\mathbf{p})}\right) e^{-\beta E_{i}^{-}(\mathbf{p})}+e^{-3 \beta E_{i}^{-}(\mathbf{p})}\right] \\
& \left.+\frac{1}{\beta} \ln \left[1+3\left(\Phi^{*}+\Phi e^{-\beta E_{i}^{+}(\mathbf{p})}\right) e^{-\beta E_{i}^{+}(\mathbf{p})}+e^{-3 \beta E_{i}^{+}(\mathbf{p})}\right]\right] \\
& +G_{\mathrm{s}}\left[\sigma^{2}+\pi^{2}\right]+3 G_{8}\left(\sigma^{2}+\pi^{2}\right)^{2}+\mathcal{U}
\end{aligned}
$$

with $E_{ \pm}^{ \pm}(\mathrm{p})=E_{ \pm}(\mathrm{p}) \pm \mu_{\mathrm{q}}$, where

$$
E_{ \pm}(\mathrm{p})=\sqrt{\left(E(\mathrm{p}) \pm \mu_{\mathrm{I}}\right)^{2}+N^{2}}
$$

for $E(\mathrm{p})=\sqrt{\mathbf{p}^{2}+M^{2}}$. On the right-hand side of (13), only the first term diverges, and it is then regularized by the threedimensional momentum cutoff $\Lambda[14,18]$. 
We use $\mathcal{U}$ of Ref. [19] that is fitted to LQCD data in the pure gauge theory at finite $T$ [50, 51]:

$$
\begin{aligned}
& \mathcal{U}= T^{4}\left[-\frac{a(T)}{2} \Phi^{*} \Phi\right. \\
&\left.+b(T) \ln \left(1-6 \Phi \Phi^{*}+4\left(\Phi^{3}+\Phi^{* 3}\right)-3\left(\Phi \Phi^{*}\right)^{2}\right)\right] \\
& a(T)=a_{0}+a_{1}\left(\frac{T_{0}}{T}\right)+a_{2}\left(\frac{T_{0}}{T}\right)^{2}, \quad b(T)=b_{3}\left(\frac{T_{0}}{T}\right)^{3}
\end{aligned}
$$

where parameters are summarized in Table \. The Polyakov potential yields a first-order deconfinement phase transition at $T=T_{0}$ in the pure gauge theory. The original value of $T_{0}$ is $270 \mathrm{MeV}$ determined from the pure gauge LQCD data, but the PNJL model with this value of $T_{0}$ yields a larger value of the pseudocritical temperature $T_{\mathrm{c}}$ at zero chemical potential than $T_{\mathrm{c}}=173 \pm 8 \mathrm{MeV}$ that the full LQCD simulation [5254] predicts. Therefore, we reset $T_{0}$ to $212 \mathrm{MeV}$ [32] so as to reproduce the LQCD result.

\begin{tabular}{cccc}
\hline \hline$a_{0}$ & $a_{1}$ & $a_{2}$ & $b_{3}$ \\
\hline 3.51 & -2.47 & 15.2 & -1.75 \\
\hline \hline
\end{tabular}

TABLE I: Summary of the parameter set in the Polyakov-potential sector determined in Ref. [19]. All parameters are dimensionless.

The classical variables $X=\Phi, \Phi^{*}, \sigma$, and $\pi$ are determined by the stationary conditions

$$
\partial \Omega / \partial X=0
$$

The solutions to the stationary conditions do not give the global minimum of $\Omega$ necessarily. There is a possibility that they yield a local minimum or even a maximum. We then have checked that the solutions yield the global minimum when the solutions $X\left(T, \mu_{\mathrm{q}}, \mu_{\mathrm{I}}\right)$ are inserted into (13).

In this work, first-order transitions are defined by (approximate) order parameters, $\sigma, \pi$ and $\Phi$ in their discontinuities. When the susceptibility of one of the order parameters diverges, we regard it as a second-order transition of the order parameter. For crossover, the pseudocritical point is determined by a peak of the susceptibility. When the susceptibility has two peaks and it is not clear which peak should be taken, we do not plot a phase boundary to avoid the confusion.

Table II shows parameters in the NJL sector used in the present analyses. As shown in Ref. [32], set A can reproduce not only the pion decay constant $f_{\pi}=93.3 \mathrm{MeV}$ and the pion mass $M_{\pi}=138 \mathrm{MeV}$ at vacuum $\left(T=\mu_{\mathrm{q}}=\mu_{\mathrm{I}}=0\right)$ but also $T_{\mathrm{c}}=173 \pm 8 \mathrm{MeV}[52-54]$ at finite temperature ( $T>0$ and $\mu_{\mathrm{q}}=\mu_{\mathrm{I}}=0$ ). For this reason, we take this parameter set in this paper. For comparison, we also use set B with no scalar-type eight-quark interaction. This parameter set also reproduces the pion mass and the pion decay constant correctly, but not LQCD data at finite temperature $\left(T>0\right.$ and $\left.\mu_{\mathrm{q}}=\mu_{\mathrm{I}}=0\right)$. The sigma-meson mass $M_{\sigma}$ is 526 (680) $[\mathrm{MeV}]$ for set A (B).

\begin{tabular}{ccccc}
\hline \hline Set & $G_{s}$ & $G_{8}$ & $m_{0}$ & $\Lambda$ \\
\hline A & $4.673\left[\mathrm{GeV}^{-2}\right]$ & $452.12\left[\mathrm{GeV}^{-8}\right]$ & $5.5[\mathrm{MeV}]$ & $631.5[\mathrm{MeV}]$ \\
B & $5.498\left[\mathrm{GeV}^{-2}\right]$ & 0 & $5.5[\mathrm{MeV}]$ & $631.5[\mathrm{MeV}]$ \\
\hline \hline
\end{tabular}

TABLE II: Summary of parameters in the NJL sector. Here, $T_{0}=$ $212 \mathrm{MeV}$ for both the sets.

\section{NUMERICAL RESULTS}

The phase structure in the $\mu_{\mathrm{I}}-\mu_{\mathrm{q}}-T$ space is explored by the PNJL model with the eight-quark interaction.

\section{A. Phase structure in the $\mu_{\mathrm{I}}-T$ plane at $\mu_{\mathrm{q}}=0$}

LQCD data are available in the $\mu_{\mathrm{I}}-T$ plane at $\mu_{\mathrm{q}}=0$ [37], since LQCD has no sign problem there. In QCD, it is known [36] that at zero $T$, a second-order phase transition occurs at $\mu_{\mathrm{I}}=M_{\pi} / 2$ from the normal $(\pi=0)$ to the pionsuperfluidity phase $(\pi \neq 0)$; this will be understood in subsection IIIB also by using the PNJL model with the eightquark interaction. The critical chemical potential $\mu_{\mathrm{c}}$ of the pion-superfluidity phase transition is $\mu_{\mathrm{c}}=0.57 / \mathrm{a}$ in LQCD calculation with a lattice spacing $a$, while it is $\mu_{\mathrm{c}}=M_{\pi} / 2=$ $69[\mathrm{MeV}]$ in the PNJL calculation. In the LQCD data, $\mu_{\mathrm{I}}$ is then normalized as $\mu_{\mathrm{c}}=69[\mathrm{MeV}]$. This makes it possible to compare the PNJL calculation with the LQCD data.

First, we consider the normal phase by taking a case of $\mu_{\mathrm{I}}=0.96 \mu_{\mathrm{c}}=66[\mathrm{MeV}]$. Figure 1 presents $\sigma$ and $\Phi$ as a function of $T / T_{\mathrm{c}}$, where $\sigma$ is normalized by the value $\sigma_{0}$ at zero $T$. LQCD data are plotted by plus $(+)$ symbols with $10 \%$ error bar; LQCD data of Refs. [37] have only small errors, but we have added $10 \%$ error that comes from LQCD data [53] on the pseudocritical temperature $T_{\mathrm{c}}$ at zero quark and isospin-chemical potentials. The PNJL result with the scalar-type eight-quark interaction (the thick-solid curve) is consistent with the LQCD data; note that the present model has no free parameter. If the scalar-type eight-quark interaction is switched off from the PNJL model, the result (the thin-solid curve) deviates sizably from the LQCD data particularly on $\sigma$. This indicates that the scalar-type eight-quark interaction is inevitable.

We use the dimensionless susceptibility matrix [15, 26]

$$
\tilde{\chi}=C^{-1}
$$

defined by the dimensionless curvature matrix

$$
\begin{aligned}
C & =\left(\begin{array}{llll}
c_{\pi \pi} & c_{\pi \sigma} & c_{\pi \Phi} & c_{\pi \bar{\Phi}} \\
c_{\sigma \pi} & c_{\sigma \sigma} & c_{\sigma \Phi} & c_{\sigma \bar{\Phi}} \\
c_{\Phi \pi} & c_{\Phi \sigma} & c_{\Phi \Phi} & c_{\Phi \bar{\Phi}} \\
c_{\bar{\Phi} \pi} & c_{\bar{\Phi} \sigma} & c_{\bar{\Phi} \Phi} & c_{\bar{\Phi} \bar{\Phi}}
\end{array}\right) \\
& =\left(\begin{array}{cccc}
T^{2} \Omega_{\pi \pi} & T^{2} \Omega_{\pi \sigma} & T^{-1} \Omega_{\pi \Phi} & T^{-1} \Omega_{\pi \bar{\Phi}} \\
T^{2} \Omega_{\sigma \pi} & T^{2} \Omega_{\sigma \sigma} & T^{-1} \Omega_{\sigma \Phi} & T^{-1} \Omega_{\sigma \bar{\Phi}} \\
T^{-1} \Omega_{\Phi \pi} & T^{-1} \Omega_{\Phi \sigma} & T^{-4} \Omega_{\Phi \Phi} & T^{-4} \Omega_{\Phi \bar{\Phi}} \\
T^{-1} \Omega_{\bar{\Phi} \pi} & T^{-1} \Omega_{\bar{\Phi} \sigma} & T^{-4} \Omega_{\bar{\Phi} \Phi} & T^{-4} \Omega_{\bar{\Phi} \bar{\Phi}}
\end{array}\right),
\end{aligned}
$$



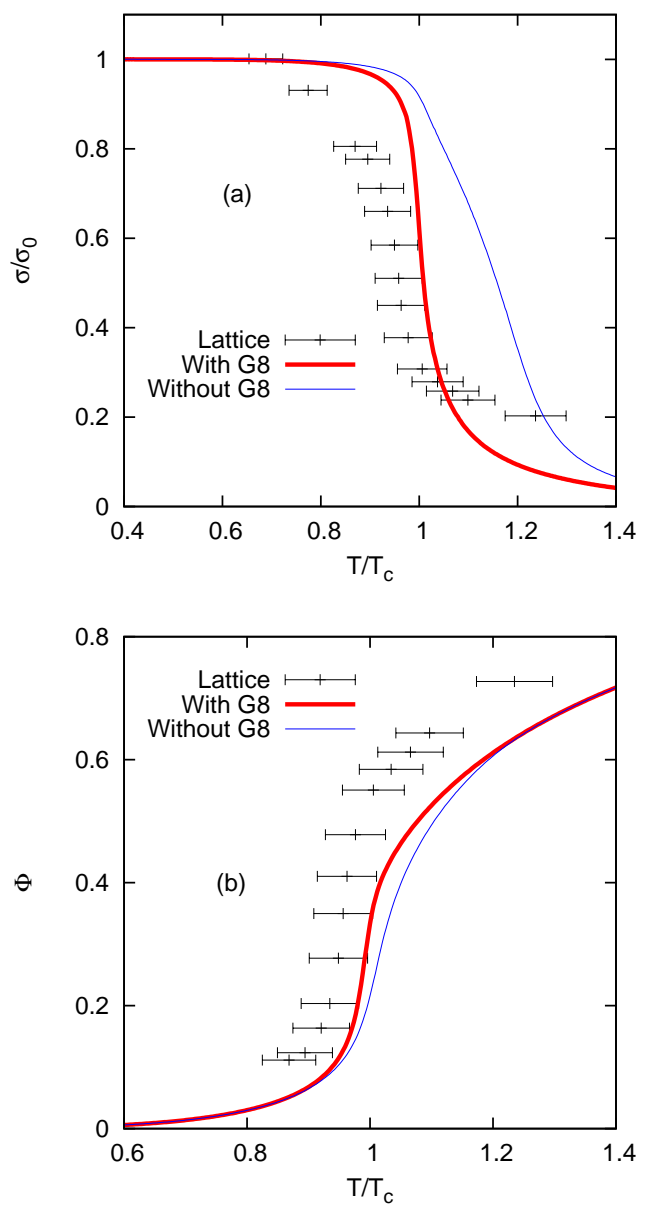

Fig. 1: (color online). $T$ dependence of (a) chiral condensate $\sigma$ and (b) Polyakov-loop $\Phi$ at $\mu_{\mathrm{I}}=0.96 \mu_{\mathrm{c}}=66[\mathrm{MeV}]$ and $\mu_{\mathrm{q}}=0$. Here, $\sigma$ is normalized by the value $\sigma_{0}$ at vacuum and $T$ is also normalized by $T_{c}=173[\mathrm{MeV}]$. The thick (thin) solid curves represent the PNJL results with (without) the scalar-type eight-quark interaction; Lattice data (+) are taken from Ref. [37]. The lattice data are plotted with 10 $\%$ error bar, since lattice calculations have $10 \%$ error in determining $T_{\mathrm{c}}[53]$.

where $\Omega_{x y}=\partial^{2} \Omega / \partial x \partial y$ for $x, y=\sigma, \pi, \Phi, \bar{\Phi}$. The susceptibilities thus defined are dimensionless. For simplicity, we take the following shorthand notation: $\tilde{\chi}_{\sigma}=\tilde{\chi}_{\sigma \sigma}, \tilde{\chi}_{\pi}=$ $\tilde{\chi}_{\pi \pi}, \tilde{\chi}_{\Phi}=\tilde{\chi}_{\Phi \Phi}$.

Figure 2 presents the chiral and the Polyakov-loop susceptibility, $\tilde{\chi}_{\sigma}$ and $\tilde{\chi}_{\Phi}$, as a function of $T$. The PNJL model with the scalar-type eight-quark interaction (the thick-solid curve) gives a better agreement with the LQCD data than the PNJL model without the scalar-type eight-quark interaction (the thin-solid curve). The present analysis for finite $\mu_{\mathrm{I}}$ is parameter free. Therefore, the reasonable agreement between the PNJL model with the eight-quark interaction and the LQCD data indicates that the PNJL model with the eightquark interaction is reliable.

Next, we consider the pion-superfluidity phase by taking a case of $\mu_{\mathrm{I}}=1.4 \mu_{\mathrm{c}}=96[\mathrm{MeV}]$. Figure 3 presents $\Phi$ and
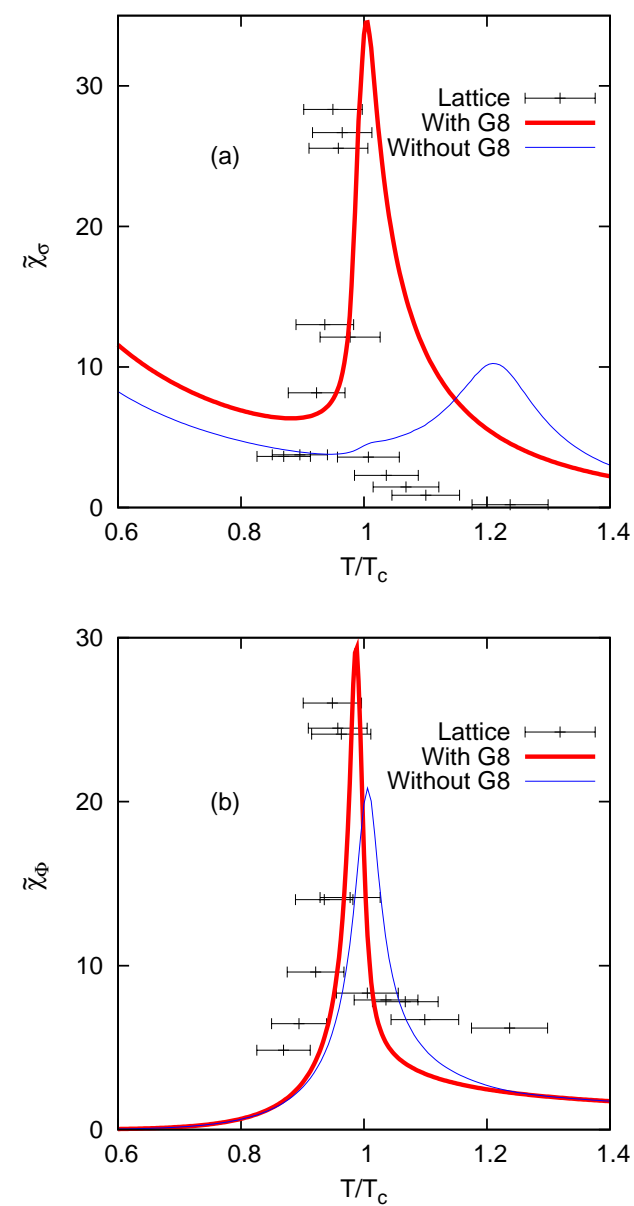

Fig. 2: (color online). $T$ dependence of (a) chiral and (b) Polyakovloop susceptibility at $\mu_{\mathrm{I}}=0.96 \mu_{\mathrm{c}}=66[\mathrm{MeV}]$ and $\mu_{\mathrm{q}}=0$. See Fig. 11 for the definition of lines and LQCD data. Since the susceptibilities of LQCD are obtained in arbitrary units, the magnitudes are then rescaled to fit the corresponding thick-solid curves, respectively.

$\pi$ as a function of $T / T_{\mathrm{c}}$, where $\pi$ is normalized by the value $\pi_{0}$ at zero $T$. Again, the PNJL model with the scalar-type eight-quark interaction (the thick-solid curve) is consistent with the LQCD data compared with the PNJL model without the scalar-type eight-quark interaction (the thin-solid curve). The PNJL calculation on $\pi$ shows that the pion-superfluidity phase transition is of second order there.

Thus, the PNJL model with the scalar-type eight-quark interaction is consistent with the LQCD data, indicating that the model is more reliable than the original PNJL model without the eight-quark interaction. Figure 4 shows the phase diagram in the $\mu_{\mathrm{I}}-T$ plane at $\mu_{\mathrm{q}}=0$. Panels (a) and (b) present results of the PNJL calculations with and without the eight-quark interaction, respectively. The thick-solid curve shows a firstorder pion-superfluidity phase transition, while the dashed line indicates a second-order pion-superfluidity phase transition. A meeting point between the two lines is a tricritical point (TCP) by definition. The dot-dashed (dotted) line stands 

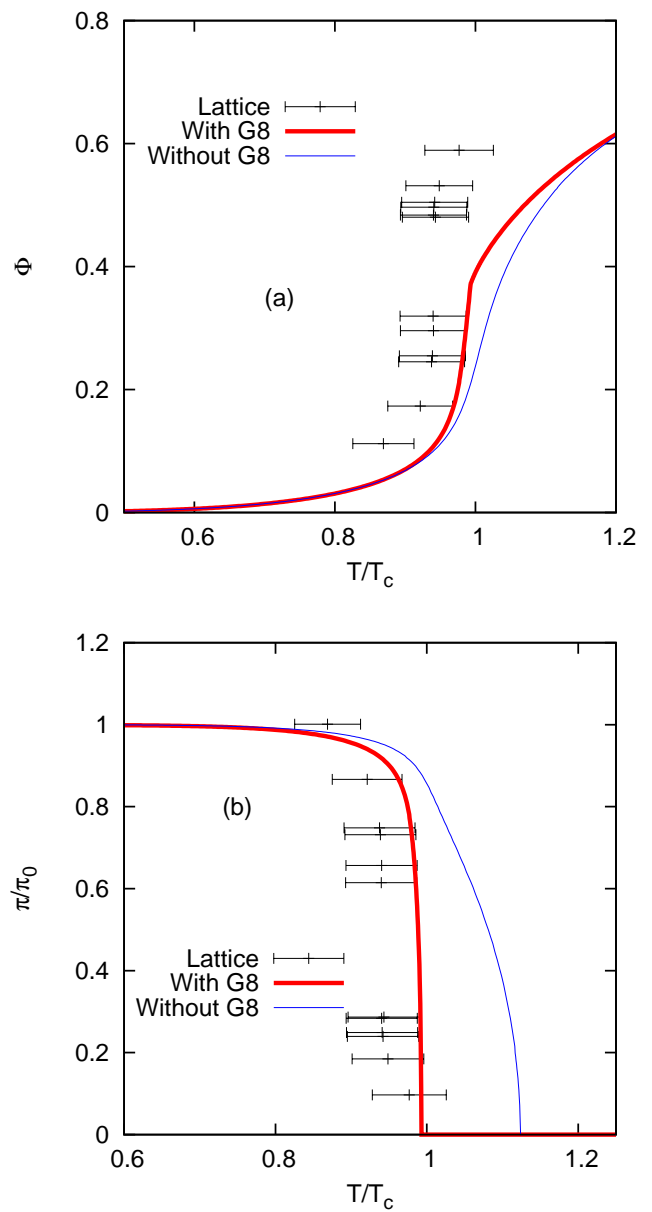

Fig. 3: (color online). $T$ dependence of (a) Polyakov-loop and (b) pion condensate at $\mu_{\mathrm{I}}=1.4 \mu_{\mathrm{c}}=96[\mathrm{MeV}]$ and $\mu_{\mathrm{q}}=0$. Here, $\pi$ is normalized by the value $\pi_{0}$ at zero $T$. See Fig. 1 for the definition of lines and the LQCD data.
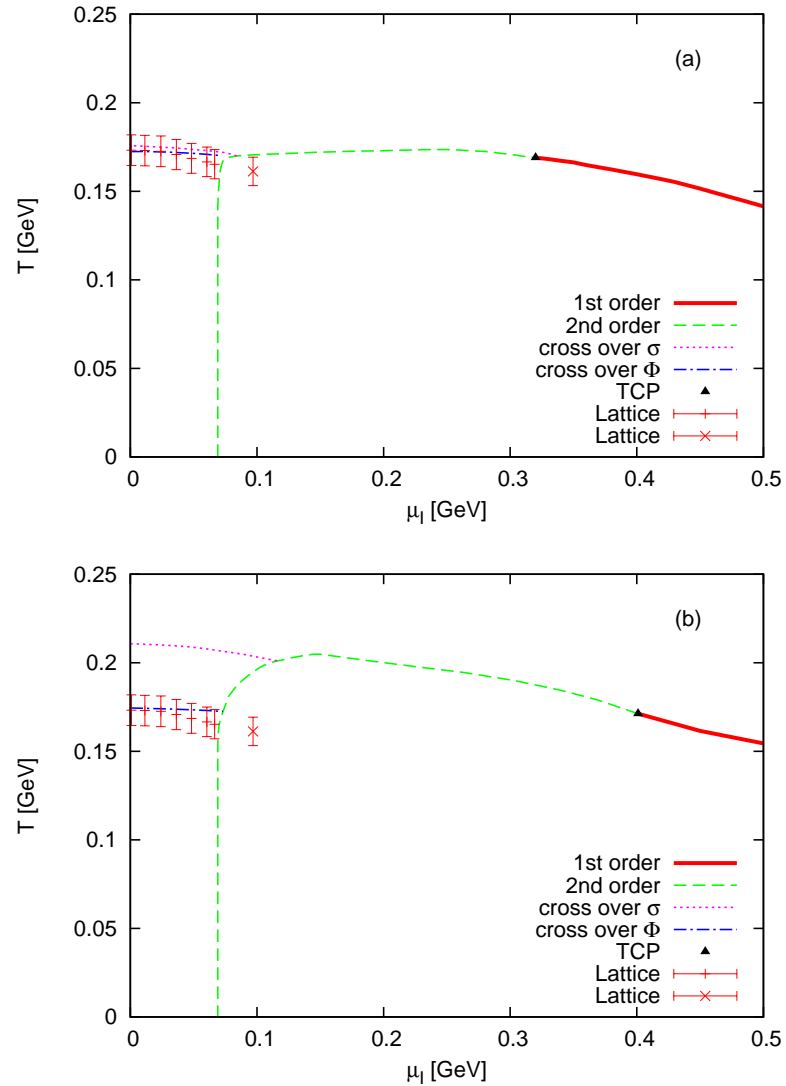

Fig. 4: (color online). Phase diagram in the $\mu_{\mathrm{I}}-T$ plane at $\mu_{\mathrm{q}}=0$ for the case (a) with and (b) without the scalar-type eight-quark interaction. The thick-solid (dashed) curve represents a first-order (second-order) pion-superfluidity phase transition. The dot-dashed (dotted) line means a deconfinement (chiral) crossover transition. At $\mu_{I}>M_{\pi} / 2, \tilde{\chi}_{\Phi}$ has two peaks, so we do not plot any deconfinement crossover transition line there. Meanwhile, the chiral crossover transition line (dotted line) terminates at TCP. LQCD on the chiral and deconfinement crossover transitions are represented by a plus $(+)$ symbol, while LQCD on the second-order pion-superfluidity transition and the deconfinement crossover transition are shown by a cross $(\times)$ symbol. See Fig. 1 for more information on LQCD data.

\section{B. Phase structure in the $\mu_{\mathrm{I}}-\mu_{\mathrm{q}}$ plane at $T=0$}

In the $\mu_{\mathrm{I}}-\mu_{\mathrm{q}}$ plane at $T=0$, the thermodynamic potential of the PNJL model is reduced to that of the NJL model:

$$
\begin{aligned}
\Omega= & -6 \sum_{i= \pm} \int \frac{d^{3} \mathrm{p}}{(2 \pi)^{3}}\left[E_{i}(\mathrm{p})-\left(E_{i}-\mu_{q}\right) \theta\left(\mu_{q}-E_{i}\right)\right] \\
& +G_{\mathrm{s}}\left[\sigma^{2}+\pi^{2}\right]+3 G_{8}\left(\sigma^{2}+\pi^{2}\right)^{2} .
\end{aligned}
$$

When $\mu_{\mathrm{I}} \leq M_{\pi} / 2, \pi=0$ and $M \approx 330 \mathrm{MeV}$, so that $E_{ \pm}=$ $E \pm \mu_{\mathrm{I}} \geq M-\frac{M_{\pi}}{2} \approx 260 \mathrm{MeV}$. Hence, when $\mu_{\mathrm{q}}<260 \mathrm{MeV}$, $\Omega$ is reduced to

$$
\Omega=G_{\mathrm{s}} \sigma^{2}+3 G_{8} \sigma^{4}-12 \int \frac{d^{3} \mathrm{p}}{(2 \pi)^{3}} E(\mathrm{p}) .
$$


Therefore, $\Omega$ does not depend on $\mu_{q}$ and $\mu_{\mathrm{I}}$ for $\mu_{\mathrm{I}}<M_{\pi} / 2$ and $\mu_{\mathrm{q}}<260 \mathrm{MeV}$, indicating that no phase transition occurs there. In other words, there is a possibility that a chiral phase transition takes place when $\mu_{\mathrm{q}}>260 \mathrm{MeV}$. This is realized, as shown later in Fig. 6. For $\mu_{\mathrm{I}}=M_{\pi} / 2$, more careful discussion is necessary, since it is a boundary of the normal phase in which $\pi=0$. This is discussed below.

In the normal-phase region at $\mu_{\mathrm{q}}<260 \mathrm{MeV}$, the curvature of $\Omega$ in the $\pi$-direction is obtained by [12]

$$
\frac{\partial^{2} \Omega}{\partial \pi^{2}}=2 G_{\pi}-48 G_{\pi}^{2} \int \frac{d^{3} \mathbf{p}}{(2 \pi)^{3}} \frac{E(\mathbf{p})}{E(\mathbf{p})^{2}-\mu_{\mathrm{I}}^{2}} \equiv f\left(\mu_{\mathrm{I}}\right),
$$

with

$$
G_{\pi}=-\frac{1}{2} \frac{\partial N}{\partial \pi} .
$$

Thus, $f\left(\mu_{\mathrm{I}}\right)$ does not depend on $\mu_{q}$. Here, an effect of the eight-quark interaction appears only through $M$ and $G_{\pi}$.

In vacuum ( $\left.T=\mu_{\mathrm{q}}=\mu_{\mathrm{I}}=0\right)$, the RPA function with external momentum $\left(q_{0} \neq 0, \mathbf{q}=0\right)$ is [11, 12, 27]

$$
2 G_{\pi}-48 G_{\pi}^{2} \int \frac{d^{3} \mathbf{p}}{(2 \pi)^{3}} \frac{E(\mathbf{p})}{E(\mathbf{p})^{2}-q_{0}^{2} / 4}=f\left(\frac{q_{0}}{2}\right),
$$

and the pion mass $M_{\pi}$ is determined by the condition $f\left(M_{\pi} / 2\right)=0$. We then find for $\mu_{\mathrm{I}}=M_{\pi} / 2$ that

$$
\frac{\partial^{2} \Omega}{\partial \pi^{2}}=f\left(\frac{M_{\pi}}{2}\right)=0 .
$$

Thus, the curvature of $\Omega$ at $\mu_{\mathrm{I}}=M_{\pi} / 2$ is zero in the $\pi$ directions, indicating that a second-order pion-superfluidity phase transition takes place at $\mu_{\mathrm{I}}=M_{\pi} / 2$ when $\mu_{\mathrm{q}}<260 \mathrm{MeV}$. This point will be confirmed later in the phase diagram of Fig. 6(a) where the second-order pion-superfluidity phase transition line (dashed line) is a straight line.

Next we consider both regions of $\mu_{\mathrm{I}} \leq M_{\pi} / 2$ and $\mu_{\mathrm{I}}>$ $M_{\pi} / 2$. Figure 5 shows $|\pi|,|\sigma|$ and $R=\sqrt{M^{2}+N^{2}}$ as a function of $\mu_{\mathrm{I}}$ and $\mu_{\mathrm{q}}$. The pion condensate $\pi$ is zero at $\mu_{\mathrm{I}}<M_{\pi} / 2$, but nonzero at $\mu_{\mathrm{I}}>M_{\pi} / 2$, as expected. Therefore, the former region is the normal $\left(I_{3}\right.$-symmetric) phase and the latter region is the pion-superfluidity $\left(I_{3}\right.$-symmetry broken) phase. The order parameter $|\sigma|$ of the chiral symmetry is almost constant in the normal phase but goes down in the pion-superfluidity phase. The parameter $R$ is almost constant over the two phases, when $\mu_{\mathrm{q}}<200 \mathrm{MeV}$. When $\mu_{\mathrm{q}}>200 \mathrm{MeV}, R$ has a discontinuity in the $\mu_{\mathrm{q}}$ direction. Thus, the $\mu_{\mathrm{q}}$ dependence of $R$ at finite $\mu_{\mathrm{I}}$ is similar to that at $\mu_{\mathrm{I}}=0$ over a wide range of $\mu_{\mathrm{I}}$.

In the limit of $m_{0}=0$ and $\mu_{\mathrm{I}}=0$, the chiral symmetry is an exact symmetry. In this situation, the thermodynamic potential of (13) is a function of $R$, and $R$ is a function of $\sqrt{\sigma^{2}+\pi^{2}}$. This means that $R$ or $\sqrt{\sigma^{2}+\pi^{2}}$ is an order parameter of the chiral symmetry. When $m_{0}$ and/or $\mu_{\mathrm{I}}$ is finite, the chiral symmetry is not an exact symmetry anymore. However, the fact that the $\mu_{\mathrm{q}}$ dependence of $R$ at finite $\mu_{\mathrm{I}}$ is similar to that at $\mu_{\mathrm{I}}=0$ means that the chiral symmetry is preserved with good accuracy. This is understood as follows.
Over the normal and pion-superfluidity phases, we have

$$
\begin{aligned}
E_{ \pm} & =\sqrt{\left(E \pm \mu_{\mathrm{I}}\right)^{2}+N^{2}} \\
& =\sqrt{p^{2}+R^{2} \pm 2 E \mu_{\mathrm{I}}+\mu_{\mathrm{I}}^{2}} .
\end{aligned}
$$

As shown in Fig. 5, $R$ is about $330 \mathrm{MeV}$ at $\mu_{\mathrm{q}} \lesssim 200 \mathrm{MeV}$ and $\mu_{\mathrm{I}}<\Lambda=631.5 \mathrm{MeV}$. In the region, $E_{ \pm}$is well approximated by $\sqrt{p^{2}+R^{2}+\mu_{\mathrm{I}}^{2}}$, because $p^{2}+R^{2}+\mu_{\mathrm{I}}^{2} \gg 2 E \mu_{\mathrm{I}}$. When $\mu_{\mathrm{q}} \gtrsim 200 \mathrm{MeV}, R$ is small and hence $E_{ \pm}$is approximated by $\sqrt{p^{2}+\mu_{\mathrm{I}}^{2}}$. Therefore, $\Omega$ is a function of $R$ or $\sqrt{\sigma^{2}+\pi^{2}}$ with good accuracy; here, note that $R \approx \sqrt{\sigma^{2}+\pi^{2}}$ because of $m_{0} \ll R$. Thus, when $T$ is small, the thermodynamics at finite $\mu_{\mathrm{q}}$ and $\mu_{\mathrm{I}}$ is controlled by an approximate order parameter $R$ of the chiral symmetry over both the normal $(\pi=0)$ and the pion-superfluidity $(\pi \neq 0)$ phase; the chiral symmetry is spontaneously broken when $R$ is finite, while it is restored when $R$ is zero. When $T \gtrsim T_{c}, R$ is not large any more. Hence, $\sigma$ and $\pi$ work independently there, as shown later in subsection IIID.

Figure 6 presents the phase diagram in the $\mu_{\mathrm{I}}-\mu_{\mathrm{q}}$ plane at $T=0$. When $T=0$, the system is in the confinement phase because $\Phi=0$ there. So we consider the chiral and pionsuperfluidity transitions only here. On the solid line, the firstorder chiral and pion-superfluidity transitions coexist, while on the dot-dashed line only the first-order chiral transition takes place. The dashed line represents the second-order pionsuperfluidity transition. In panel (a) where the eight-quark interaction is taken into account, the solid, dot-dashed and dashed lines meet at a point. This is a TCP, because the pionsuperfluidity transition changes the order from first order to second order there, while the chiral transition keeps first order. Thus, there is no CEP in the $\mu_{\mathrm{I}}-\mu_{\mathrm{q}}$ plane at $T=0$. In panel (b) where the eight-quark interaction is switched off, the endpoint of the dot-dashed line is a CEP and a meeting point of the solid and dashed lines is a TCP by definition. Comparing the two panels, we can see that the eight-quark interaction changes the phase diagram qualitatively.

Recently, it was shown in Ref. [55] that the $\Lambda$ dependence of $\Omega$ may change the order of the phase transition in the mean field level. We then investigate the $\Lambda$ dependence of the phase diagram in the $\mu_{\mathrm{I}}-\mu_{\mathrm{q}}$ plane at $T=0$. In this procedure, we consider the four-quark and eight-quark interactions only. The parameters of the PNJL model are determined for each $\Lambda$ so as to reproduce the pion decay constant $f_{\pi}=93.3 \mathrm{MeV}$ and the pion mass $M_{\pi}=138 \mathrm{MeV}$ at vacuum and $T_{\mathrm{c}}=173 \pm 8 \mathrm{MeV}$ at finite temperature with no $\mu_{\mathrm{q}}$ and $\mu_{\mathrm{I}}[52-54]$; note that $T_{\mathrm{c}}$ is a much stronger constraint on $G_{8}$ than $M_{\sigma}$, since $M_{\sigma}$ has a large error bar [32]. This parameter fitting is exactly the same as that in Sec. It to determine the parameter set A.

We vary $\Lambda$ from 573 to $651.5 \mathrm{MeV}$. The upper and the lower bound of $\Lambda$ are determined as follows. The QCD sum rule yields the lower and the upper bound of $|\sigma|$ as $|\sigma|=$ $(225 \pm 25 \mathrm{MeV})^{3}$ [56, 57]. The absolute value of the chiral condensate, $|\sigma|$, increases as $\Lambda$ goes up, and reaches the upper bound of $|\sigma|$ when $\Lambda=651.5 \mathrm{MeV}$. Thus, $\Lambda=651.5 \mathrm{MeV}$ is the upper bound of $\Lambda$. Meanwhile, the lower bound of $\Lambda$ is determined by not the lower bound of $|\sigma|$ but the fact 
that no parameter set can reproduce $f_{\pi}=93.3 \mathrm{MeV}$ and $M_{\pi}=138 \mathrm{MeV}$ simultaneously when $\Lambda<573 \mathrm{MeV}$. Although this fact is found by numerical calculations, it can be understood with reasonable approximations. At zero temperature, the thermodynamic potential of the PNJL model is reduced to that of the NJL model, as shown in (20). In the NJL model, the pion mass is obtained by

$$
M_{\pi}^{2}=\frac{-4 m_{0} \sigma}{\left(M-m_{0}\right) M I\left(M, M_{\pi}\right)}
$$

with

$$
I\left(M, M_{\pi}\right)=\frac{8 N_{f} N_{c}}{2 \pi^{2}} \int_{0}^{\Lambda} \frac{p^{2} d p}{\sqrt{p^{2}+M^{2}}\left[4\left(p^{2}+M^{2}\right)-M_{\pi}^{2}\right]},
$$

where $N_{f}$ and $N_{c}$ are the numbers of colors and flavors, respectively, and $N_{f}=2$ and $N_{c}=3$ in the present case. Since $M \gg m_{0}$ in (27), and $M^{2} \gg M_{\pi}^{2}$ in (28), we then neglect $m_{0}$ in (27) and $M_{\pi}$ in (28) in order to understand the mathematical structure of (27) and (28). Using the approximate equations and the Gell-Mann-Oakes-Renner relation, we have

$$
\frac{N_{f} N_{c}}{4 \pi^{2}} x^{2}\left[\ln \frac{1+\sqrt{1+x^{2}}}{x}-\frac{1}{\sqrt{1+x^{2}}}\right]=\frac{f_{\pi}^{2}}{\Lambda^{2}},
$$

where $x=M / \Lambda$. The left-hand side of (29) has a maximum at $x=0.97$, while the right-hand side increases monotonously as $\Lambda$ goes down. This means that there exists a lower bound of $\Lambda$ that satisfies (29). The lower bound is $\Lambda=573 \mathrm{MeV}$, although $\sigma$ obtained there is within the constraint $|\sigma|=(225 \pm 25 \mathrm{MeV})^{3}$ from the QCD sum rule.

Table III presents three parameter sets, A, A' and A”, obtained by the above procedure. Set $\mathrm{A}$ is the original parameter set mentioned in Sec. [II set A' is an example of the parameter sets near the lower bound of $\Lambda$, and set $\mathrm{A}$ " is the parameter set at the upper bound of $\Lambda$. The value of $\Lambda$ in set $A$ " is slightly larger than that in set A. This indicates that set A" yields qualitatively the same phase diagram as set A. Actually, we have confirmed this with numerical calculations. Meanwhile, the phase diagram calculated with set $A^{\prime}$ is shown in Fig. 7. The phase structure shows no qualitative difference from the result of set A in Fig. [6), although the first-order chiral transition line (dot-dashed line) and the pion-superfluidity phasetransition line (solid line) are slightly shifted down by decreasing $\Lambda$. Furthermore, we have confirmed that the phase diagram does not change qualitatively near the lower bound. Thus, the order of the phase-transition is not changed by varying $\Lambda$ in the range $573<\Lambda<651.5 \mathrm{MeV}$. As a property of the parameter sets near the lower bound of $\Lambda, G_{8}$ is quite large. This means that the higher-order multiquark interactions than the eight-quark interaction may not be negligible there. However, this sort of analyses is beyond the scope of the present work.

\section{Phase structure in the $\mu_{\mathrm{q}}-T$ plane at $\mu_{\mathrm{I}}=0$}

The phase diagram in the $\mu_{\mathrm{q}}-T$ plane at $\mu_{\mathrm{I}}=0$ is shown in Fig. 8. The solid curve shows a coexistence line of first-

\begin{tabular}{lcccc}
\hline \hline Set & $G_{s}$ & $G_{8}$ & $m_{0}$ & $\Lambda$ \\
\hline A' & $5.755\left[\mathrm{GeV}^{-2}\right]$ & $1264.2\left[\mathrm{GeV}^{-8}\right]$ & $5.77[\mathrm{MeV}]$ & $580[\mathrm{MeV}]$ \\
A & $4.673\left[\mathrm{GeV}^{-2}\right]$ & $452.12\left[\mathrm{GeV}^{-8}\right]$ & $5.5[\mathrm{MeV}]$ & $631.5[\mathrm{MeV}]$ \\
A” & $4.295\left[\mathrm{GeV}^{-2}\right]$ & $351.32\left[\mathrm{GeV}^{-8}\right]$ & $5.31[\mathrm{MeV}]$ & $651.5[\mathrm{MeV}]$ \\
\hline \hline
\end{tabular}

TABLE III: Cutoff dependence of parameters. Here, $T_{0}=203 \mathrm{MeV}$ for the set $\mathrm{A}^{\prime}, T_{0}=212 \mathrm{MeV}$ for the set $\mathrm{A}$ and $T_{0}=217 \mathrm{MeV}$ for the set A".

order chiral and deconfinement phase transitions that ends at $\left(\mu_{\mathrm{q}}, T\right)=(178[\mathrm{MeV}], 152[\mathrm{MeV}])$. This point is a CEP by definition and is known to be of second-order [8, 10]. In general, once a first-order phase transition takes place for some order parameter, the discontinuity propagates to other order parameters unless the parameters are zero [33, 58]. The coexistence between the first-order chiral and deconfinement phase transitions shown in Fig. 8 is a typical case of the coexistence theorem. The dot-dashed (dotted) line stands for a crossover deconfinement (chiral) transition. The crossover chiral and deconfinement transitions almost coincide with each other and end at the CEP. Thus, a CEP exists in the present model. This CEP survives, even if the eight-quark interaction is switched off. In the case of no eight-quark interaction, the CEP in the $\mu_{\mathrm{q}}-T$ plane at $\mu_{\mathrm{I}}=0$ moves to a CEP in the $\mu_{\mathrm{I}}-\mu_{\mathrm{q}}$ plane at $T=0$ of Fig. 6(b), as $\mu_{\mathrm{I}}$ increases from zero. This behavior of CEP is changed a lot by the eight-quark interaction, as shown later in Fig. 10

In principle, the Polyakov-potential $\mathcal{U}$ depends on $\mu_{\mathrm{q}}$ as a consequence of the backreaction of the Fermion sector to the gluon sector. Particularly, the $\mu_{\mathrm{q}}$ dependence of the parameter $T_{0}$ in $\mathcal{U}$ is important and estimated by using renormalization group arguments [22]:

$$
T_{0}\left(\mu_{\mathrm{q}}\right)=T_{\tau} e^{-\frac{1}{\alpha_{0} b\left(\mu_{\mathrm{q}}\right)}}
$$

for $b\left(\mu_{\mathrm{q}}\right)=29 /(6 \pi)-32 \mu_{\mathrm{q}}^{2} /\left(\pi T_{\tau}^{2}\right)$ with $\alpha_{0}=0.304$ and $T_{\tau}=1.770[\mathrm{GeV}]$. Figure 9 shows effects of $T_{0}\left(\mu_{\mathrm{q}}\right)$ on the phase diagram in the $\mu_{\mathrm{q}}-T$ plane at $\mu_{\mathrm{I}}=0$. Comparing this figure with Fig. 8, we can see that the effect dose not yield any qualitative change, but the location of CEP is moved from $\left(\mu_{\mathrm{q}}, T\right)=(178[\mathrm{MeV}], 152[\mathrm{MeV}])$ to $\left(\mu_{\mathrm{q}}, T\right)=$ (187 [MeV], $130[\mathrm{MeV}])$. At small $T$, the effect becomes negligible, since $\mathcal{U}$ itself tends to zero as $T$ decreases.

\section{Phase structure in the $\mu_{\mathrm{I}}-\mu_{\mathrm{q}}-T$ space}

Figure 10 presents the phase diagram in the $\mu_{\mathrm{I}}-\mu_{\mathrm{q}}-T$ space. In this space, TCP and CEP emerge not at points but on lines; precisely speaking, CEP appears on lines CD and DA, while TCP does on lines GD and DA. Thus, CEP moves from point $\mathrm{C}$ to $\mathrm{A}$ via $\mathrm{D}$ as $\mu_{\mathrm{I}}$ increases from zero. Meanwhile, TCP moves from point $\mathrm{A}$ to $\mathrm{G}$ via $\mathrm{D}$ as $\mu_{\mathrm{q}}$ increases from zero.

Line $\mathrm{GE}$ is a second-order pion-superfluidity transition line in the $\mu_{\mathrm{I}}-\mu_{\mathrm{q}}$ plane at $T=0$. A track of the line with respect to increasing $T$ becomes an area GEAD. Hence, the 

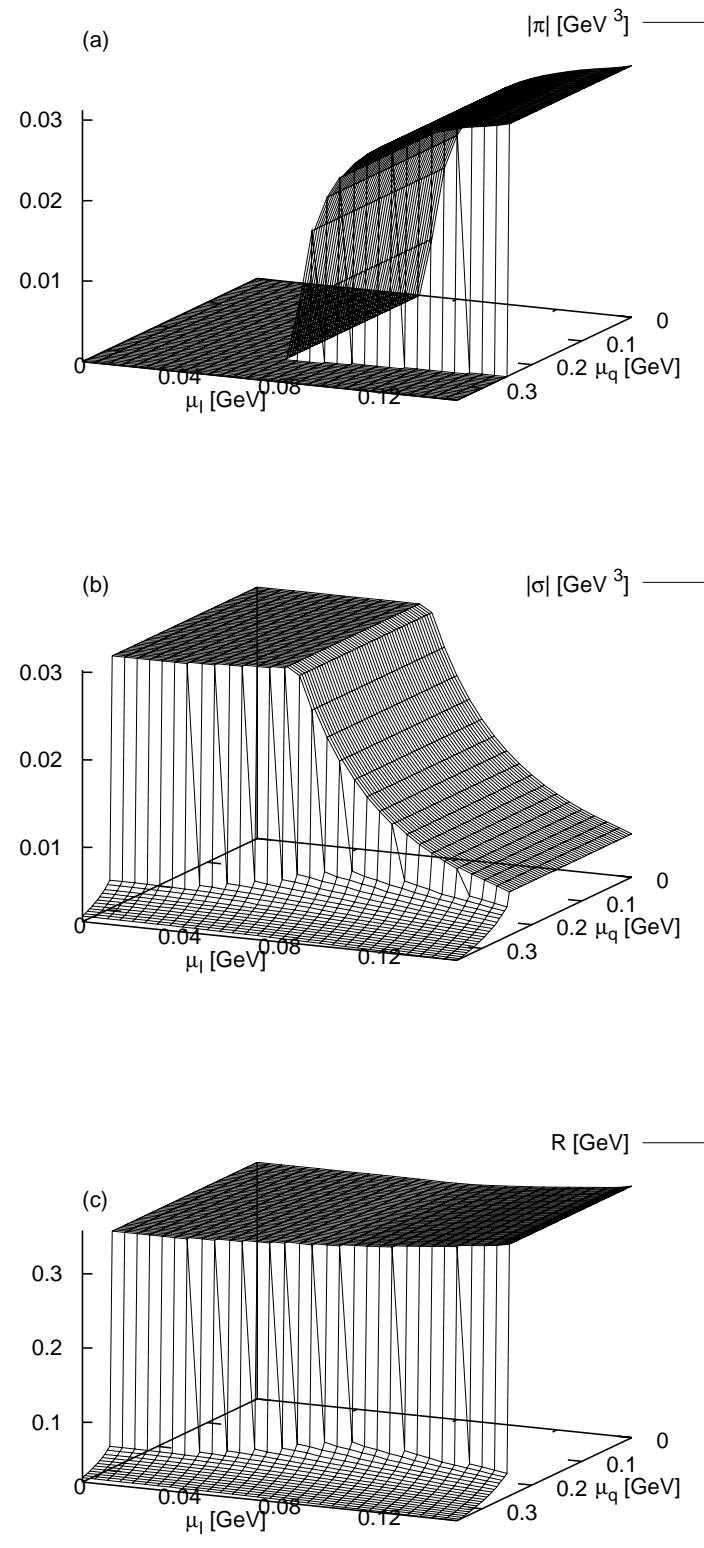

Fig. 5: (color online). Order parameters (a) $\pi$, (b) $\sigma$ and (c) $R$ as a function of $\mu_{\mathrm{I}}$ and $\mu_{\mathrm{q}}$.

pion-superfluidity transition is second order on the area. Similarly, a track of line FG $(\mathrm{GH})$ with respect to increasing $T$ becomes an area FGDC (GHBAD). In area FGDC, the chiral and deconfinement transitions are of first order, while the pion condensate is zero. In area GHBAD, all the chiral, deconfinement and pion-superfluidity transitions are of first order. The two areas smoothly connect to each other, indicating that the thermodynamics in these areas are controlled by $R$. Properties of lines and areas in Fig. [10 are summarized in Table [V] while locations of points in Fig. 10 are summarized in Table $\mathrm{V}$.
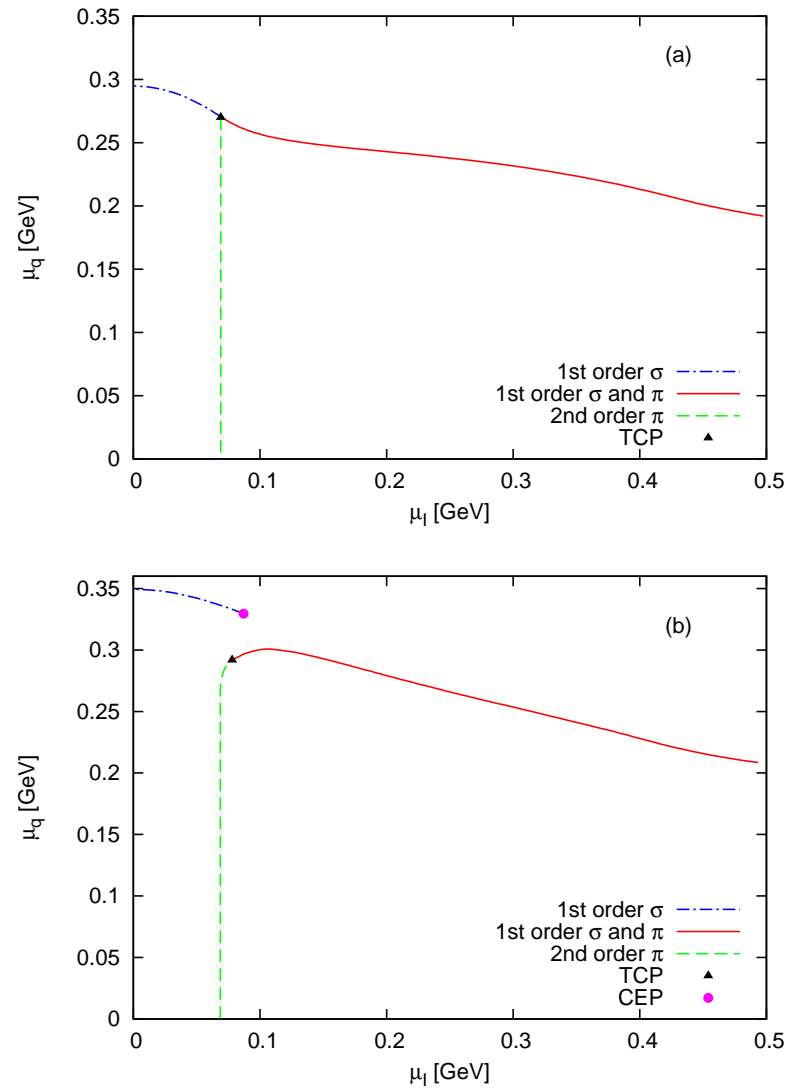

Fig. 6: (color online). Phase diagram in the $\mu_{\mathrm{I}}-\mu_{\mathrm{q}}$ plane at $T=$ 0 for the case (a) with and (b) without the eight-quark interaction. The solid line represents a coexistence line of first-order chiral and pion-superfluidity phase transitions, while the dot-dashed line shows a first-order chiral phase-transition line. The dashed line stands for a second-order pion-superfluidity phase transition.

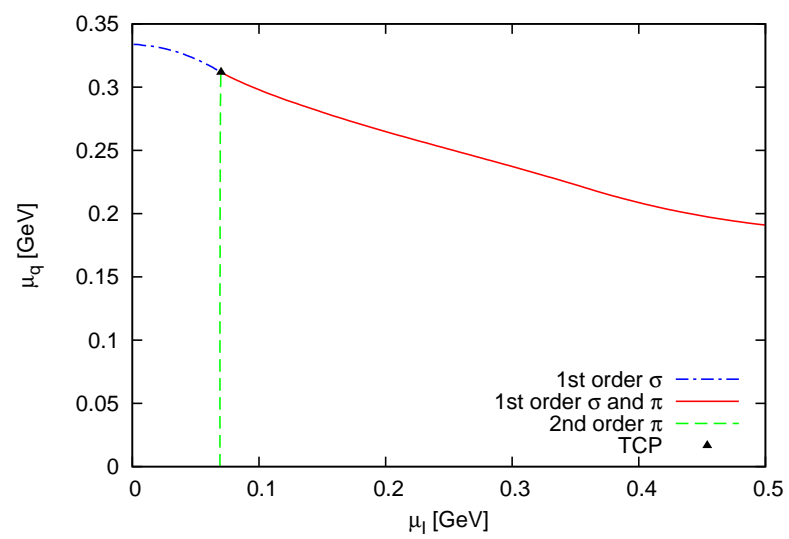

Fig. 7: (color online). Phase diagram in the $\mu_{\mathrm{I}}-\mu_{\mathrm{q}}$ plane at $T=0$ calculated with set A'. See Fig. 6 for the definition of lines. 


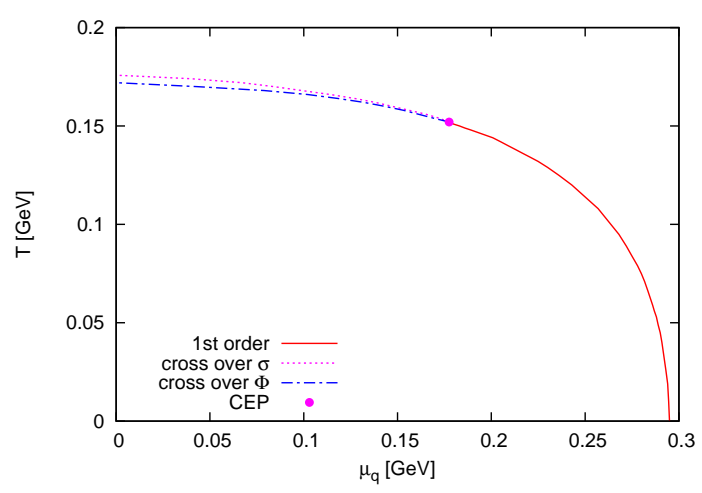

Fig. 8: (color online). Phase diagram in the $\mu_{\mathrm{q}}-T$ plane at $\mu_{\mathrm{I}}=0$. The solid line is a coexistence line of first-order chiral and deconfinement phase transitions. The dashed line stands for the chiral crossover transition, while the dot-dashed line does for the deconfinement crossover transition. Here, the eight-quark interaction is taken into account in the PNJL model.

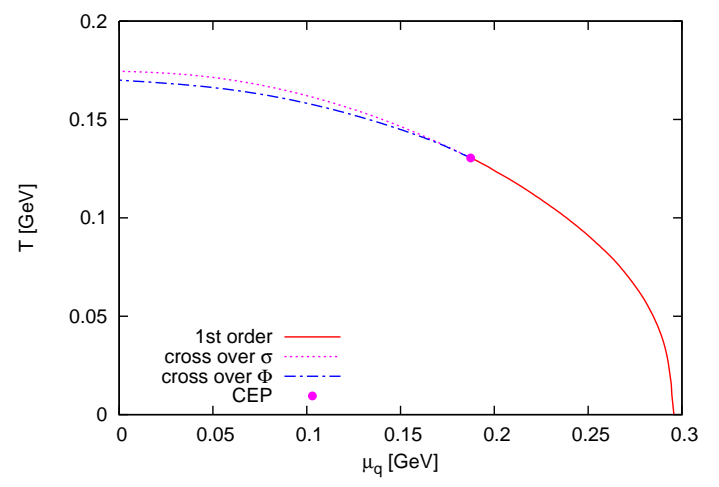

Fig. 9: (color online). Effect of $\mu_{\mathrm{q}}$-dependent $T_{0}$ on the phase diagram in the $\mu_{\mathrm{q}}-T$ plane at $\mu_{\mathrm{I}}=0$. In the PNJL calculation with the eight-quark interaction, parameter $T_{0}$ is replaced by $\mu_{\mathrm{q}}$-dependent parameter $T_{0}\left(\mu_{\mathrm{q}}\right)$. See Fig. 8 for the definition of lines.

Figure 11 presents the chiral susceptibility $\tilde{\chi}_{\sigma}$, the Polyakov-loop susceptibility $\tilde{\chi}_{\Phi}$ and the pion susceptibility $\tilde{\chi}_{\pi}$ as a function of $\mu_{\mathrm{q}}$ for the case of $\left(\mu_{\mathrm{I}}, T\right)=$ $(0.075[\mathrm{GeV}], 0.140[\mathrm{GeV}])$; these are plotted by the solid, dashed and dotted curves, respectively. The $\mu_{\mathrm{q}}$ dependence of these susceptibilities correspond to a line parallel to the $\mu_{\mathrm{q}}$ axis in Fig. 10. The susceptibilities $\tilde{\chi}_{\sigma}$ and $\tilde{\chi}_{\Phi}$ have peaks at the same position $\mu_{\mathrm{q}}=187 \mathrm{MeV}$, indicating that the chiral and deconfinement transitions are second order there. This position corresponds to a point on line CD in Fig. 10. Meanwhile, $\tilde{\chi}_{\pi}$ has a peak at $\mu_{\mathrm{q}}=173 \mathrm{MeV}$. This second-order critical point of the pion-superfluidity transition corresponds to a point on area ADGE in Fig. 10 As an interesting feature, $\tilde{\chi}_{\sigma}$ is discontinuous at $\mu_{\mathrm{q}}=173 \mathrm{MeV}$. This property will be analyzed in Sec. IIIE

Figure 12 shows $\tilde{\chi}_{\sigma}, \tilde{\chi}_{\Phi}$ and $\tilde{\chi}_{\pi}$ as a function of $\mu_{\mathrm{q}}$ for the case of $\left(\mu_{\mathrm{I}}, T\right)=(0.100[\mathrm{GeV}], 0.169[\mathrm{GeV}])$. All the suscep-

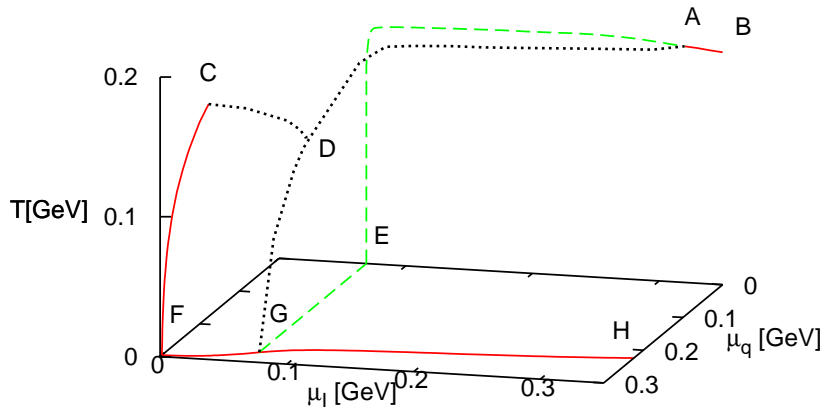

Fig. 10: (color online). Phase diagram in the $\mu_{\mathrm{I}}-\mu_{\mathrm{q}}-T$ space. Here, the eight-quark interaction is taken into account in the PNJL model. Properties of lines and areas are summarized in Table [V] while locations of points are summarized in Table $\mathrm{V}$

\begin{tabular}{cccc}
\hline \hline area & $\sigma$ & $\pi$ & $\Phi$ \\
\hline CDGF & 1 st & $\pi=0$ & 1 st \\
\hline ABHGD & 1 st & 1 st & 1 st \\
\hline ADGE & \multicolumn{2}{c}{ 2nd } \\
\hline \hline & & & \\
\hline \hline line & $\sigma$ & $\pi$ & $\Phi$ \\
\hline CF & 1 st & $\pi=0$ & 1 st \\
\hline CD & CEP & $\pi=0$ & CEP \\
\hline FG & 1 st & $\pi=0$ & $\Phi=0$ \\
\hline AD & CEP & TCP & CEP \\
\hline DG & 1 st & TCP & 1 st \\
\hline GE & & 2nd & $\Phi=0$ \\
\hline EA & & 2nd & \\
\hline GH & 1 st & 1st & $\Phi=0$ \\
\hline \hline
\end{tabular}

TABLE IV: Properties of areas and lines in Fig. 10 The phrase "1st" ("2nd") means that the phase transition either in the area or on the line is first (second) order. Blank means that no significant transition takes place there.

tibilities have peaks at the same position $\mu_{\mathrm{q}}=51 \mathrm{MeV}$, indicating that chiral, deconfinement and pion-superfluidity transitions of second order take place simultaneously there. This critical point corresponds to a point on line DA in Fig. 10. This is a TCP for $\pi$ and a CEP for $\sigma$ and $\Phi$. As an interesting feature, each of $\tilde{\chi}_{\sigma}$ and $\tilde{\chi}_{\Phi}$ has a kink at $\mu_{\mathrm{q}}=51 \mathrm{MeV}$. This property will be analyzed in Sec. IIIE.

Now, the phase diagram in the $\mu_{\mathrm{I}}-\mu_{\mathrm{q}}-T$ space is understood more precisely by considering the $\mu_{\mathrm{q}}-T$ plane at four values of $\mu_{\mathrm{I}}$ : each belongs to any of four regions, (i) $\mu_{\mathrm{I}}<\mu_{\mathrm{I}}(G)=$ $M_{\pi} / 2$, (ii) $\mu_{\mathrm{I}}(G)<\mu_{\mathrm{I}}<\mu_{\mathrm{I}}(D)$, (iii) $\mu_{\mathrm{I}}(D)<\mu_{\mathrm{I}}<\mu_{\mathrm{I}}(A)$ and (iv) $\mu_{\mathrm{I}}(A)<\mu_{\mathrm{I}}$, where $\mu_{\mathrm{I}}(X)$ is a value of $\mu_{\mathrm{I}}$ at point $\mathrm{X}$. 


\begin{tabular}{|c|c|c|c|c|c|}
\hline poin & ( & $T[\mathrm{GeV}$ &,$\mu_{\mathrm{q}}[\mathrm{GeV}]$ &,$\mu_{\mathrm{I}}[\mathrm{GeV}]$ & \\
\hline A & ( & 0.169 & 0 & 0.320 & ) \\
\hline B & ( & 0.166 & 0 & 0.350 & ) \\
\hline $\mathrm{C}$ & ( & 0.152 & 0.178 & 0 & 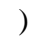 \\
\hline D & ( & 0.136 & 0.190 & 0.084 & ) \\
\hline $\mathrm{E}$ & ( & 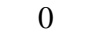 & 0 & 0.069 & ) \\
\hline $\mathrm{F}$ & ( & 0 & 0.295 & 0 & ) \\
\hline G & ( & 0 & 0.270 & 0.069 & ) \\
\hline $\mathrm{H}$ & ( & 0 & 0.223 & 0.350 & \\
\hline
\end{tabular}

TABLE V: Locations of points in Fig. 10,

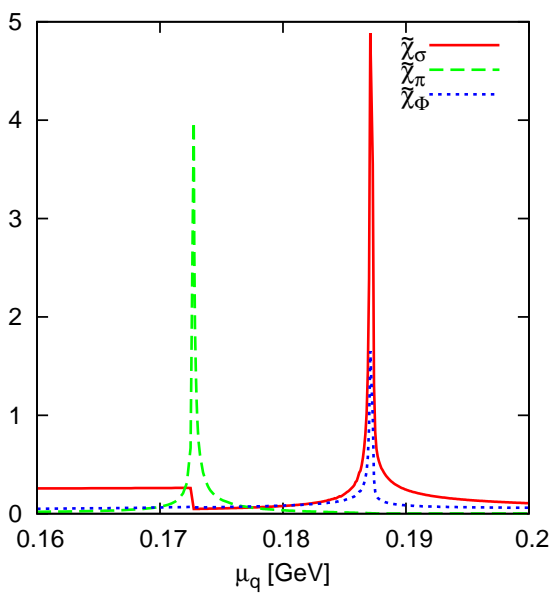

Fig. 11: (color online). Chiral, pion and Polyakov-loop susceptibilities as a function of $\mu_{\mathrm{q}}$ at $\left(\mu_{\mathrm{I}}, T\right)=(0.075[\mathrm{GeV}], 0.140[\mathrm{GeV}])$. Here, the eight-quark interaction is taken into account in the PNJL model. These are represented by the solid, dashed and dotted, respectively. See Ref. [11] for the definition of the susceptibilities. The $\tilde{\chi}_{\sigma}$ and $\tilde{\chi}_{\pi}$ are multiplied by $10^{-3}$ and $10^{-5}$, respectively, but $\tilde{\chi}_{\Phi}$ is not multiplied by any factor.

The $\mu_{\mathrm{q}}-T$ phase diagram in region (i) is essentially equal to that in the $\mu_{\mathrm{q}}-T$ plane at $\mu_{\mathrm{I}}=0$, i.e., Fig. 8] since $\pi$ is always zero there.

The $\mu_{\mathrm{q}}-T$ phase diagram in region (ii) is a bit more complicated, as shown in Figure 13 where $\mu_{I}=75 \mathrm{MeV}$ is taken as an example. In Fig. 13, the thick-solid line ending at TCP stands for a coexistence line of first-order chiral, deconfinement and pion-superfluidity transitions. This is a natural result of the coexistence theorem of the first-order phase transition [33, 58]. Meanwhile, on the thin-solid line between TCP and CEP, first-order chiral and deconfinement transitions coexist, but any first-order pion-superfluidity transition does not take place, because $\pi$ is zero above the dashed line starting from TCP that represents a second-order pion-superfluidity transition.

The $\mu_{\mathrm{q}}-T$ phase diagram in region (iii) is simpler than that in region (ii). Fig. 14 presents the $\mu_{\mathrm{q}}-T$ plane at $\mu_{I}=100 \mathrm{MeV}$ belonging to region (iii). As shown by the

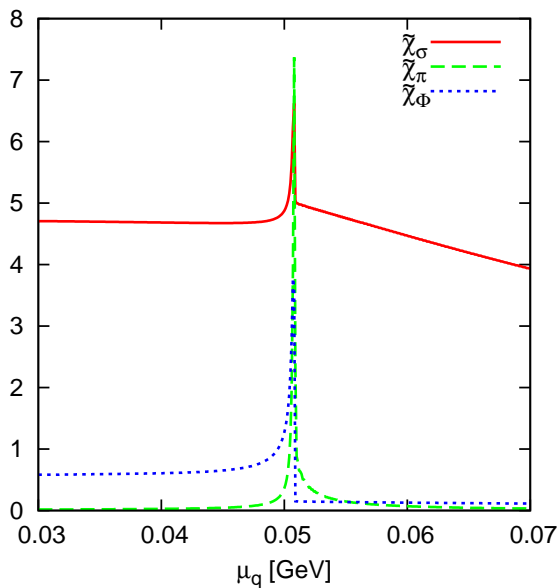

Fig. 12: (color online). Chiral, pion and Polyakov-loop susceptibilities as a function of $\mu_{\mathrm{q}}$ at $\left(\mu_{\mathrm{I}}, T\right)=(0.100[\mathrm{GeV}], 0.169[\mathrm{GeV}])$. Here, the eight-quark interaction is taken into account in the PNJL model. See Fig. 11 for the definition of lines. $\tilde{\chi}_{\sigma}$ and $\tilde{\chi}_{\pi}$ are multiplied by $1 / 20$ and $10^{-4}$, respectively, but $\tilde{\chi}_{\Phi}$ is not multiplied by any factor.

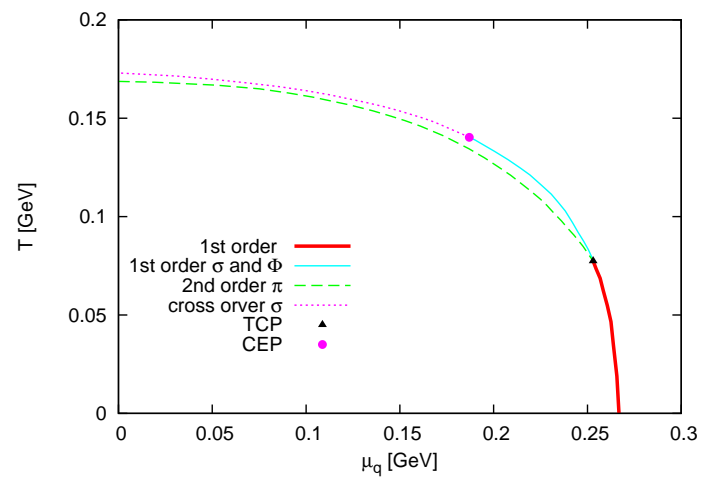

Fig. 13: (color online). Phase diagram in the $\mu_{\mathrm{q}}-T$ plane at $\mu_{\mathrm{I}}=$ $75 \mathrm{MeV}$. Here, the eight-quark interaction is taken into account in the PNJL model.

thick-solid line, all the first-order chiral, deconfinement and pion-superfluidity transitions occur simultaneously there. A second-order pion-superfluidity transition and a crossover chiral transition occur on the dashed line start from a point shown by triangle. This point is a TCP for $\pi$ and a CEP for $\sigma$ by definition. The point corresponds to a point on line DA in Fig.10.

The $\mu_{\mathrm{q}}-T$ phase diagram in region (iv) is simple and easily imaginable from Fig. 10, In this region, only a coexistence line of first-order chiral, deconfinement and pion-superfluidity transitions exists. 


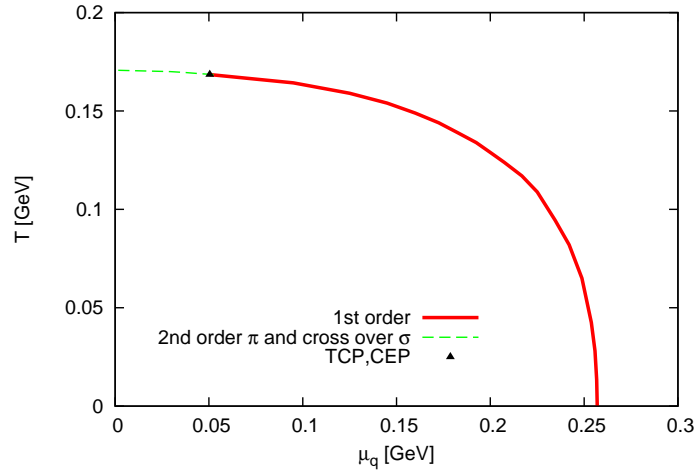

Fig. 14: (color online). Phase diagram in the $\mu_{\mathrm{q}}-T$ plane at $\mu_{\mathrm{I}}=$ $100 \mathrm{MeV}$. Here, the eight-quark interaction is taken into account in the PNJL model. The thick-solid line represents a coexistence line of the first-order chiral, deconfinement and pion-superfluidity transitions. On the dashed line, a second-order pion-superfluidity transition and a crossover chiral transition occur simultaneously.

\section{E. Properties of susceptibilities}

Properties of the susceptibilities near the second-order pion-superfluidity transition line, CEP and TCP are investigated.

For simplicity, we take the following shorthand notation for the curvature matrix $C$ of 19 :

$$
C=\left(\begin{array}{cc}
c_{\pi \pi} & A \\
A^{\mathrm{T}} & K
\end{array}\right)
$$

where $A=\left(c_{\pi \sigma}, c_{\pi \Phi}, c_{\pi \bar{\Phi}}\right), A^{\mathrm{T}}$ is the transverse of $A$, and the matrix $K$ is expressed by

$$
K=\left(\begin{array}{ccc}
c_{\sigma \sigma} & c_{\sigma \Phi} & c_{\sigma \bar{\Phi}} \\
c_{\Phi \sigma} & c_{\Phi \Phi} & c_{\Phi \bar{\Phi}} \\
c_{\bar{\Phi} \sigma} & c_{\bar{\Phi} \Phi} & c_{\sigma \bar{\Phi}}
\end{array}\right)
$$

As shown in (13), $\Omega$ is an even function of $\pi$. Noting that $c_{X Y}(X, Y=\pi, \sigma, \Phi, \bar{\Phi})$ are proportional to $\partial^{2} \Omega / \partial X \partial Y$; therefore, we can find that $c_{\pi \pi}$ and $c_{x y}$ for $x, y=\sigma, \Phi, \bar{\Phi}$ are $\pi$-even, while $c_{x \pi}$ and $c_{\pi y}$ for $x, y=\sigma, \Phi, \bar{\Phi}$ are $\pi$-odd.

First, we consider the normal $(\pi=0)$ phase including the second-order pion-superfluidity transition line. Since $\pi=0$ in this phase, the $\pi$-odd quantities $c_{x \pi}$ and $c_{\pi y}$ for $x, y=\sigma, \Phi, \bar{\Phi}$ are zero. Therefore, $C$ is reduced to

$$
C=\left(\begin{array}{cc}
c_{\pi \pi} & 0 \\
0 & K
\end{array}\right)
$$

Equation (33) shows the following properties.

1. On the second-order pion-superfluidity transition line, the curvature $c_{\pi \pi}$ in the $\pi$ direction is zero by definition of the second-order transition. Therefore, $\operatorname{det}[C]=0$. This indicates that $\tilde{\chi}_{\pi}=\operatorname{det}[K] / \operatorname{det}[C]$ diverges on the transition line, since $\operatorname{det}[K]$ is not zero in general.
2. If a CEP of the chiral phase transition appears in the normal phase, the determinant $\operatorname{det}[K]$ is zero at the CEP; see Ref.[10] for the details of this proof. Hence, $\tilde{\chi}_{\sigma}$ is divergent at the CEP because of $\operatorname{det}[C]=$ $c_{\pi \pi} \operatorname{det}[K]=0$.

Properties 1 and 2 are understood clearly with numerical results shown in Fig. 11. The peak of $\tilde{\chi}_{\pi}$ at $\mu_{\mathrm{q}}=\mu_{\mathrm{q}}^{\pi}=$ $173 \mathrm{MeV}$ shows a second-order pion-superfluidity transition, while the peak of $\tilde{\chi}_{\sigma}$ at $\mu_{\mathrm{q}}=\mu_{\mathrm{q}}^{\sigma}=187 \mathrm{MeV}$ does a CEP in the normal phase. Hence, the thermal system is in the normal phase $(\pi=0)$ for $\mu_{\mathrm{q}}>\mu_{\mathrm{q}}^{\pi}$ and in the broken phase $(\pi \neq 0)$ for $\mu_{\mathrm{q}}<\mu_{\mathrm{q}}^{\pi}$. Figure 15 shows $\operatorname{det}[C]$ and $\operatorname{det}[K]$ as a function of $\mu_{\mathrm{q}}$ at $\left(\mu_{\mathrm{I}}, T\right)=(0.075[\mathrm{GeV}], 0.140[\mathrm{GeV}])$. It is found from this figure that $\operatorname{det}[C]=0$ and $\operatorname{det}[K] \neq 0$ at $\mu_{\mathrm{q}}=\mu_{\mathrm{q}}^{\pi}$, while $\operatorname{det}[C]=\operatorname{det}[K]=0$ at $\mu_{\mathrm{q}}=\mu_{\mathrm{q}}^{\sigma}$. Thus, properties 1 and 2 are confirmed to be true by the numerical results.

Next, we consider the broken $(\pi \neq 0)$ phase. Fig. 11 is a good example. At $\mu_{\mathrm{q}}$ slightly smaller than $\mu_{\mathrm{q}}^{\pi}, \pi$ is small, because $\pi=0$ at $\mu_{\mathrm{q}}=\mu_{\mathrm{q}}^{\pi}$. Hence, any quantity can be expanded into a power series of $\pi$. After the expansion, the $\pi$-even quantities $c_{x y}(x, y=\sigma, \Phi, \bar{\Phi})$ are of order $(\pi)^{0}$, while the $\pi$-odd quantities $c_{\pi y}$ and $c_{x \pi}(x, y=\sigma, \Phi, \bar{\Phi})$ are of order $(\pi)^{1}$. The entry $c_{\pi \pi}$ is of order $(\pi)^{2}$, as shown below. The stationary condition (17) for $\pi$ is rewritten into

$$
0=\frac{\partial \Omega}{\partial \pi}=\frac{\partial \Omega}{\partial \pi^{2}} \frac{d \pi^{2}}{d \pi}=\frac{\partial \Omega}{\partial \pi^{2}} 2 \pi,
$$

and hence

$$
\frac{\partial \Omega}{\partial \pi^{2}}=0
$$

because of $\pi \neq 0$. Expanding the $\pi$-even function $\Omega$ into a power series of $\pi^{2}$,

$$
\Omega=\sum_{n} a_{n} \pi^{2 n},
$$

one can see from (35) that

$$
a_{1}=0 .
$$

Hence, $c_{\pi \pi}=T^{2} \partial^{2} \Omega / \partial \pi \partial \pi$ is of order $(\pi)^{2}$. Therefore, the matrix $C$ is the following property in the broken phase:

3. At $\mu_{\mathrm{q}}$ slightly smaller than $\mu_{\mathrm{q}}^{\pi}, c_{\pi \pi}$ is of order $(\pi)^{2}, A$ and $A^{\mathrm{T}}$ are of order $(\pi)^{1}$, and $K$ is of order $(\pi)^{0}$.

Now, we consider the reason why $\tilde{\chi}_{\sigma}$ is discontinuous at $\mu_{\mathrm{q}}=\mu_{\mathrm{q}}^{\pi}$ in Fig. 11. The susceptibility $\tilde{\chi}_{\sigma}$ is expressed by

$$
\tilde{\chi}_{\sigma}=\frac{\Delta_{\sigma \sigma}}{\operatorname{det}[C]},
$$

where $\Delta_{\sigma \sigma}$ is the cofactor of entry $c_{\sigma \sigma}$ in the matrix $C$. Property 3 indicates that both $\Delta_{\sigma \sigma}$ and $\operatorname{det}[C]$ are of order $(\pi)^{2}$ in the broken phase at $\mu_{\mathrm{q}}<\mu_{\mathrm{q}}^{\pi}$, so that the left-hand limit of $\tilde{\chi}_{\sigma}$ as $\mu_{\mathrm{q}}$ approaches $\mu_{\mathrm{q}}^{\pi}$ is finite. As an important point, the $\pi$ odd quantities contribute to this left-hand limit. Meanwhile, the $\pi$-odd quantities are zero in the normal-phase at $\mu_{\mathrm{q}}>\mu_{\mathrm{q}}^{\pi}$, 
so that they do not contribute to the right-hand limit of $\Delta_{\sigma \sigma}$ and $\operatorname{det}[C]$ as $\mu_{\mathrm{q}}$ approaches $\mu_{\mathrm{q}}^{\pi}$. Thus, the right-hand limit of $\tilde{\chi}_{\sigma}$ is different from the left-hand limit of $\tilde{\chi}_{\sigma}$.

In Fig. 12, all the susceptibilities, $\tilde{\chi}_{\sigma}, \tilde{\chi}_{\Phi}$ and $\tilde{\chi}_{\pi}$, have peaks at the same position $\mu_{\mathrm{q}}=\mu_{\mathrm{q}}^{\pi}=51 \mathrm{MeV}$. The divergence of $\tilde{\chi}_{\sigma}$ means that in (38), the $\operatorname{denominator} \operatorname{det}[C]$ tends to zero faster than the numerator $\Delta_{\sigma \sigma}$ as $\mu_{\mathrm{q}}$ approaches $\mu_{\mathrm{q}}^{\pi}$ from the left-hand side. There is no guarantee that such a strong damping of $\operatorname{det}[C]$ also happens in the right-hand limit, because $\pi$-odd quantities $c_{\sigma y}$ and $c_{x \pi}$ are zero there. Actually, such a fast damping in the right-hand limit does not occur here, as shown by the numerical calculation. As $\mu_{\mathrm{q}}$ approaches $\mu_{\mathrm{q}}^{\pi}$, therefore, $\tilde{\chi}_{\sigma}$ is divergent in the left-hand limit, but finite in the right-hand limit. This is the reason why $\tilde{\chi}_{\sigma}$ has a kink at $\mu_{\mathrm{q}}=51 \mathrm{MeV}$.

The fast damping of $\operatorname{det}[C]$ in both the left- and the righthand limit happens only on point D in Fig. 10, as shown below. Figure 16 presents $\tilde{\chi}_{\sigma}, \tilde{\chi}_{\Phi}$ and $\tilde{\chi}_{\pi}$ as a function of $\mu_{\mathrm{q}}$ at $\mu_{\mathrm{I}}=0.08425 \mathrm{GeV}$ and $T=0.136 \mathrm{GeV}$. All the susceptibilities diverge at $\mu_{\mathrm{q}}=0.190 \mathrm{GeV}$. This peak corresponds to point D in Fig. 10. In this case, obviously, the susceptibilities have no kink. Therefore, $\operatorname{det}[C]$ tends to zero faster than $\Delta_{\sigma \sigma}$ in both the right- and the left-hand limit. Point $\mathrm{D}$ is a meeting point of CEP and TCP. There is no guarantee that such a special critical point always happens. Actually, such a point does not appear if the eight-quark interaction is switched off, as shown in Fig. 17, here, line CD (AG) represents CEP (TCP) of the chiral (pion-superfluidity) phase transition and there is no meeting point between CEP and TCP.

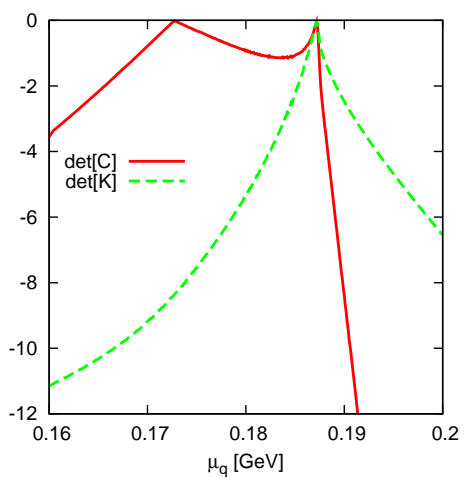

Fig. 15: (color online). $\mu_{\mathrm{q}}$ dependence of $\operatorname{det}[C]$ and $\operatorname{det}[K]$ at $T=0.14[\mathrm{GeV}]$ and $\mu_{\mathrm{I}}=0.075[\mathrm{GeV}]$. The solid (dashed) line stands for $\operatorname{det}[C](\operatorname{det}[K])$. Here, the eight-quark interaction is taken into account in the PNJL model. The $\operatorname{det}[C]$ is multiplied by $6 \times 10^{2}$.

\section{SUMMARY}

Critical points such as CEP and TCP are important as indicators of the chiral, deconfinement and pion-superfluidity phase transitions in measurements at GSI, SPS, RHIC and LHC. In the measurements, $\mu_{\mathrm{I}}$ is not zero generally. We have then predicted the phase diagram of two-flavor QCD in the

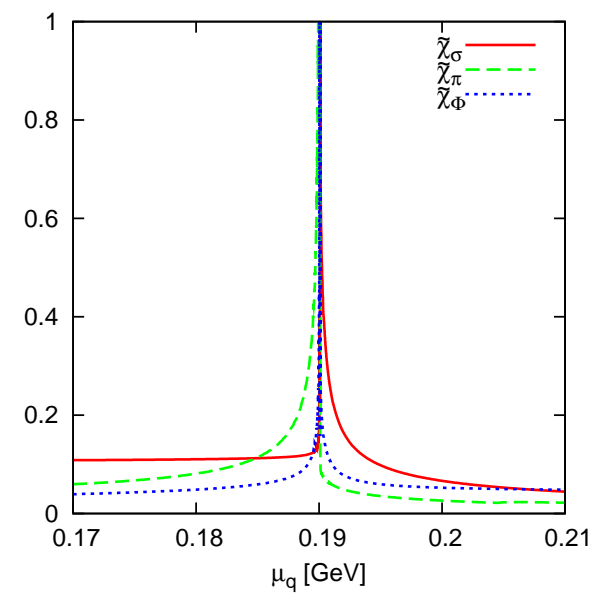

Fig. 16: (color online). Chiral, pion and Polyakov-loop susceptibilities as a function of $\mu_{\mathrm{q}}$ at $\left(\mu_{\mathrm{I}}, T\right)=(0.08425[\mathrm{GeV}], 0.136[\mathrm{GeV}])$. Here, the eight-quark interaction is taken into account in the PNJL model. See Fig. 11 for the definition of lines. The $\tilde{\chi}_{\sigma}$ and $\tilde{\chi}_{\pi}$ are multiplied by $5 \times 10^{-4}$ and $2 \times 10^{-4}$, respectively, but $\tilde{\chi}_{\Phi}$ is not multiplied by any factor.

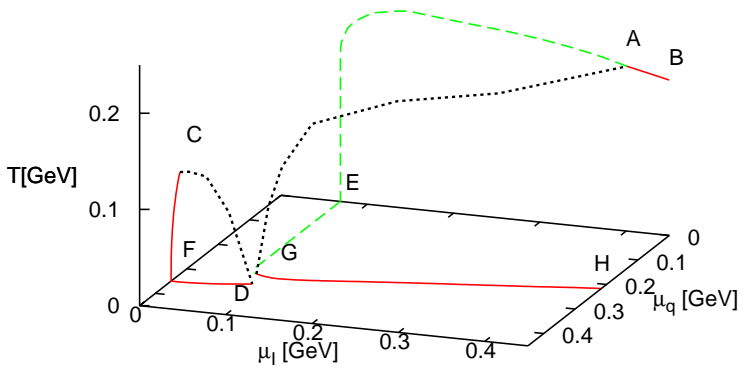

Fig. 17: (color online). Phase diagram in the $\mu_{\mathrm{I}}-\mu_{\mathrm{q}}-T$ space calculated with the PNJL model with the four-quark interaction only. See Fig.10 for the definition of lines and areas, except line CD represents CEP and line AG stands for TCP.

$\mu_{\mathrm{I}}-\mu_{\mathrm{q}}-T$ space by using the PNJL model with the scalar-type eight-quark interaction. The PNJL model with the scalar-type eight-quark interaction is consistent with the LQCD data [37] in the $\mu_{\mathrm{I}}-T$ plane at $\mu_{\mathrm{q}}=0$, while the original PNJL model without the scalar-type eight-quark interaction is not.

In the $\mu_{\mathrm{q}}-\mu_{\mathrm{I}}-T$ space, as shown in Fig.10, a CEP in the $\mu_{\mathrm{q}^{-}}$ $T$ plane at $\mu_{\mathrm{I}}=0$ moves to a TCP in the $\mu_{\mathrm{I}}-T$ plane $\mu_{\mathrm{q}}=0$ as $\mu_{\mathrm{I}}$ increases. Meanwhile, the TCP in the $\mu_{\mathrm{I}}-T$ plane at $\mu_{\mathrm{q}}=0$ moves to a TCP in the $\mu_{\mathrm{q}}-\mu_{\mathrm{I}}$ plane at $T=0$. When $\mu_{\mathrm{I}}<M_{\pi} / 2$, the pion condensate $\pi$ is zero and hence a CEP exists but any TCP does not. When $M_{\pi} / 2<\mu_{\mathrm{I}} \lesssim 80 \mathrm{MeV}$, a CEP and a TCP exist separately. And when $\mu_{\mathrm{I}} \gtrsim 80 \mathrm{MeV}$, they coexist. If the eight-quark interaction is switched off, a 
CEP in the $\mu_{\mathrm{q}}-T$ plane at $\mu_{\mathrm{I}}=0$ moves to a CEP in the $\mu_{\mathrm{q}}-\mu_{\mathrm{I}}$ plane at $T=0$ as $\mu_{\mathrm{I}}$ increases; see Fig. 17. Thus, the eightquark interaction changes the QCD diagram qualitatively in the $\mu_{\mathrm{q}}-\mu_{\mathrm{I}}-T$ space.

When $T$ is small, the thermodynamics at finite $\mu_{\mathrm{I}}$ and $\mu_{\mathrm{q}}$ is controlled by $\sqrt{\sigma^{2}+\pi^{2}}$. The quantity $\sqrt{\sigma^{2}+\pi^{2}}$ is an approximate order parameter of the chiral symmetry over the $I_{3}$ symmetric $(\pi=0)$ and $I_{3}$-symmetry broken $(\pi \neq 0)$ phases.

\section{Acknowledgments}

Authors thank P. de. Forcrand, A. Nakamura, K. Nagata and K. Kashiwa for useful discussions. H. K. also thanks M. Imachi, H. Yoneyama H. Aoki and M. Tachibana for useful discussions. Y. S acknowledges support by JSPS.
[1] J. B. Kogut and D. K. Sinclair Phys. Rev. D 77, 114503 (2008).

[2] Z. Fodor, and S. D. Katz, Phys. Lett. B 534, 87 (2002); J. High Energy Phys. 03, 014 (2002).

[3] C. R. Allton, S. Ejiri, S. J. Hands, O. Kaczmarek, F. Karsch, E. Laermann, Ch. Schmidt, and L. Scorzato, Phys. Rev. D 66, 074507 (2002); S. Ejiri, C. R. Allton, S. J. Hands, O. Kaczmarek, F. Karsch, E. Laermann, and C. Schmidt, Prog. Theor. Phys. Suppl. 153, 118 (2004).

[4] P. de Forcrand and O. Philipsen, Nucl. Phys. B642, 290 (2002); P. de Forcrand and O. Philipsen, Nucl. Phys. B673, 170 (2003).

[5] M. D'Elia and M. P. Lombardo, Phys. Rev. D 67, 014505 (2003); Phys. Rev. D 70, 074509 (2004); M. D'Elia, F. D. Renzo, and M. P. Lombardo, Phys. Rev. D 76, 114509 (2007);

[6] H. S. Chen and X. Q. Luo, Phys. Rev. D72, 034504 (2005); arXiv:hep-lat/0702025 (2007); L. K. Wu, X. Q. Luo, and H. S. Chen, Phys. Rev. D76, 034505 (2007).

[7] Y. Nambu and G. Jona-Lasinio, Phys. Rev. 122, 345 (1961); Phys. Rev. 124, 246 (1961).

[8] M. Asakawa and K. Yazaki, Nucl. Phys. A504, 668 (1989).

[9] M. Kitazawa, T. Koide, T. Kunihiro, and Y. Nemoto, Prog. Theor. Phys. 108, 929 (2002).

[10] H. Fujii, Phys. Rev. D 67, 094018 (2003); H. Fujii, and M. Ohtani, Phys. Rev. D 70, 014016 (2004).

[11] K. Kashiwa, H. Kouno, T. Sakaguchi, M. Matsuzaki, and M. Yahiro, Phys. Lett. B 647, 446 (2007); K. Kashiwa, M. Matsuzaki, H. Kouno, and M. Yahiro, Phys. Lett. B 657, 143 (2007); T. Sakaguchi, K. Kashiwa, M. Matsuzaki, H. Kouno, and M. Yahiro, Centr. Eur. J. Phys. 6, 116 (2008).

[12] L. He, M. Jin, and P. Zhuang, Phys. Rev. D 71, 116001 (2005).

[13] P. N. Meisinger, and M. C. Ogilvie, Phys. Lett. B 379, 163 (1996).

[14] K. Fukushima, Phys. Lett. B 591, 277 (2004).

[15] K. Fukushima, Phys. Rev. D 77, 114028 (2008); Phys. Rev. D 78, 114019 (2008); Phys. Rev. D 79, 074015 (2009);

[16] S. K. Ghosh, T. K. Mukherjee, M. G. Mustafa, and R. Ray, Phys. Rev. D 73, 114007 (2006).

[17] E. Megías, E. R. Arriola, and L. L. Salcedo, Phys. Rev. D 74, 065005 (2006).

[18] C. Ratti, M. A. Thaler, and W. Weise, Phys. Rev. D 73, 014019 (2006); C. Ratti, S. Rößner, M. A. Thaler, and W. Weise, Eur. Phys. J. C 49, 213 (2007).

[19] S. Rößner, C. Ratti, and W. Weise, Phys. Rev. D 75, 034007 (2007).

[20] M. Ciminale, R. Gatto, N. D. Ippolito, G. Nardulli, and M. Ruggieri, Phys. Rev. D 77, 054023 (2008); M. Ciminale, G. Nardulli, M. Ruggieri, and R. Gatto, Phys. Lett. B 657, 64 (2007).

[21] C. Sasaki, B. Friman, and K. Redlich, Phys. Rev. D 75, 074013 (2007).

[22] B. -J. Schaefer, J. M. Pawlowski, and J. Wambach, Phys. Rev. D 76, 074023 (2007).
[23] Z. Zhang, and Y. -X. Liu, Phys. Rev. C 75, 064910 (2007).

[24] S. Mukherjee, M. G. Mustafa, and R. Ray, Phys. Rev. D 75, 094015 (2007).

[25] H. Hansen, W. M. Alberico, A. Beraudo, A. Molinari, M. Nardi, and C. Ratti, Phys. Rev. D 75, 065004 (2007); P. Costa, C. A. de Sousa, M. C. Ruivo, and H. Hansen, Europhys. Lett. 86, 31001 (2009); P. Costa, M. C. Ruivo, C. A. de Sousa, H. Hansen, and W. M. Alberico, Phys. Rev. D 79, 116003 (2009).

[26] K. Kashiwa, H. Kouno, M. Matsuzaki, and M. Yahiro, Phys. Lett. B 662, 26 (2008).

[27] W. J. Fu, Z. Zhang, and Y. X. Liu, Phys. Rev. D 77, 014006 (2008); Phys. Rev. D 79, 074011 (2009);

[28] H. Abuki, M. Ciminale, R. Gatto, G. Nardulli, and M. Ruggieri, Phys. Rev. D 77, 074018 (2008); H. Abuki, M. Ciminale, R. Gatto, N. D. Ippolito, G. Nardulli, and M. Ruggieri, Phys. Rev. D 78, 014002 (2008); H. Abuki, R. Anglani, R. Gatto, and G. Nardulli, Phys. Rev. D 78, 034034 (2008); H. Abuki, Prog. Theor. Phys. Suppl. 174, 66 (2008); H. Abuki, M. Ciminale, R. Gatto, and M. Ruggieri, Phys. Rev. D 79, 034021 (2009).

[29] S. Rößner, T. Hell, C. Ratti, and W. Weise, Nucl. Phys. A814, 118 (2008); T. Hell, S. Rößner, M. Cristoforetti, and W. Weise, Phys. Rev. D 79, 014022 (2009).

[30] H. Abuki and K. Fukushima, Phys. Lett. B 676, 57 (2009).

[31] Y. Sakai, K. Kashiwa, H. Kouno, and M. Yahiro, Phys. Rev. D 77, 051901(R) (2008); Phys. Rev. D 78, 036001 (2008); Y. Sakai, K. Kashiwa, H. Kouno, M. Matsuzaki, and M. Yahiro, Phys. Rev. D 78, 076007 (2008); K. Kashiwa, M. Matsuzaki, H. Kouno, Y. Sakai, and M. Yahiro, Phys. Rev. D 79, 076008 (2009); H. Kouno, Y. Sakai, K. Kashiwa, and M. Yahiro, J. Phys. G: Nucl. Part. Phys. 36, 115010 (2009).

[32] Y. Sakai, K. Kashiwa, H. Kouno, M. Matsuzaki, and M. Yahiro, Phys. Rev. D 79, 096001 (2009).

[33] K. Kashiwa, M. Yahiro, H. Kouno, M. Matsuzaki, and Y. Sakai, J. Phys. G: Nucl. Part. Phys. 36, 10501 (2009);

[34] B.-J. Schaefer and M. Wagner, Phys. Rev. D 79, 014018 (2009).

[35] A. Roberge and N. Weiss, Nucl. Phys. B275, 734 (1986).

[36] D. T. Son and M. A. Stephanov, Phys. Rev. Lett. 86, 592 (2001).

[37] J. B. Kogut and D. K. Sinclair, Phys. Rev. D70, 094501(2004).

[38] P. Cea, L. Cosmai, M. D'Elia, C. Manneschi, and A. Papa, Phys. Rev. D 80, 034501 (2009).

[39] M. D'Elia and F. Sanfilippo, Phys. Rev. D 80, 014502 (2009)

[40] Y. Sakai, H. Kouno, and M. Yahiro, J. Phys. G:Nucl. Part. Phys. 37, 105007 (2010).

[41] Y. Nishida, Phys. Rev. D 69, 094501 (2004).

[42] L.F. Palhares, E.S. Fraga, and C. Villavicencio, Nucl. Phys. A820, 287c (2009); E.S. Fraga, L.F. Palhares, and C. Villavicencio, Phys. Rev. D 79, 014021 (2009).

[43] T. Anticic et al., arXiv:0909.0485[hep-ex](2009).

[44] T. Anticic et al., Phys. Rev. C 81, 064907 (2010). 
[45] R. A.Lacey et al., Phys. Rev. Lett. 98, 092301 (2007).

[46] P. Sorensen, arXiv:nucl-ex/0701028(2007).

[47] T. Nayak, arXiv:0904.3428[hep-ex](2009).

[48] A. Bhattacharyya, P. Deb, S. K. Ghosh and R. Ray, Phys. Rev. D 82, 014021 (2010).

[49] A.A. Osipov B. Hiller, and J. da Providência, Phys. Lett. B 634, 48 (2006); A.A. Osipov B. Hiller, A.H. Blin, and J. da Providência, Ann. Phys. (N.Y.) 322, 2021 (2007); A.A. Osipov B. Hiller, J. Moreira, A.H. Blin, and J. da Providência, Phys. Lett. B 659, 270 (2008); A.A. Osipov B. Hiller, J. Moreira, A.H. Blin, and J. da Providência, Phys. Lett. B 646, 91 (2007); A.A. Osipov B. Hiller, A.H. Blin, and J. da Providência, Phys. Lett. B 650, 262 (2007); B. Hiller, J. Moreira, A.A. Osipov, and A.H. Blin, Phys. Rev. D 81, 116005 (2010); J. Moreira, B. Hiller, A.A. Osipov, and A.H. Blin, arXiv:hep-ph/1008.0569 (2010).

[50] G. Boyd, J. Engels, F. Karsch, E. Laermann, C. Legeland,
M. Lütgemeier, and B. Petersson, Nucl. Phys. B469, 419 (1996).

[51] O. Kaczmarek, F. Karsch, P. Petreczky, and F. Zantow, Phys. Lett. B 543, 41 (2002).

[52] F. Karsch, Lect. notes Phys. 583, 209 (2002).

[53] F. Karsch, E. Laermann, and A. Peikert, Nucl. Phys. B 605, 579 (2001).

[54] M. Kaczmarek and F. Zantow, Phys. Rev. D 71, 114510 (2005).

[55] V. Skokov B. Friman E. Nakano K. Redlich and B.-J. Schaefer, Phys. Rev. D 82, 034029 (2010).

[56] J. Gasser, and H. Leutwyler, Phys. Rep. 87, 77 (1982).

[57] L.J. Reinders, H. Rubinstein, and S. Yazaki, Phys. Rep. 127, 1 (1985).

[58] A. Barducci, R. Casalbuoni, G. Pettini, and R. Gatto, Phys. Lett. B 301, 95 (1993). 